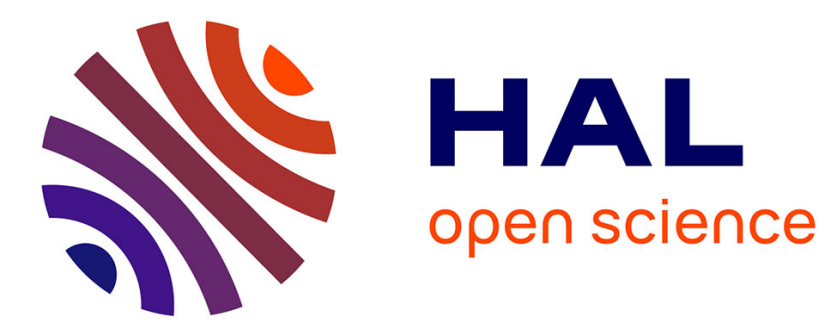

\title{
An automatic key discovery approach for data linking
}

\author{
Nathalie Pernelle, Fatiha Saïs, Danai Symeonidou
}

\section{To cite this version:}

Nathalie Pernelle, Fatiha Saïs, Danai Symeonidou. An automatic key discovery approach for data linking. Journal of Web Semantics, 2013, 23, pp.16-30. 10.1016/j.websem.2013.07.001 . hal-01276599

\section{HAL Id: hal-01276599 \\ https://hal.inria.fr/hal-01276599}

Submitted on 19 Feb 2016

HAL is a multi-disciplinary open access archive for the deposit and dissemination of scientific research documents, whether they are published or not. The documents may come from teaching and research institutions in France or abroad, or from public or private research centers.
L'archive ouverte pluridisciplinaire HAL, est destinée au dépôt et à la diffusion de documents scientifiques de niveau recherche, publiés ou non, émanant des établissements d'enseignement et de recherche français ou étrangers, des laboratoires publics ou privés. 


\title{
An Automatic Key Discovery Approach for Data Linking
}

\author{
Nathalie Pernelle, Fatiha Saïs, Danai Symeonidou \\ LRI, Paris Sud University, Bât. 650, F-91405 Orsay, FRANCE
}

\begin{abstract}
In the context of Linked Data, different kinds of semantic links can be established between data. However when data sources are huge, detecting such links manually is not feasible. One of the most important types of links, the identity link, expresses that different identifiers refer to the same real world entity. Some automatic data linking approaches use keys to infer identity links, nevertheless this kind of knowledge is rarely available. In this work we propose $\mathrm{KD} 2 \mathrm{R}$, an approach which allows the automatic discovery of composite keys in RDF data sources that may conform to different schemas. We only consider data sources for which the Unique Name Assumption is fulfilled. The obtained keys are correct with respect to the RDF data sources in which they are discovered. The proposed algorithm is scalable since it allows the key discovery without having to scan all the data. KD2R has been tested on real datasets of the international contest OAEI 2010 and on data sets available on the web of data, and has obtained promising results.
\end{abstract}

Keywords: Data Linking, Identity Links, keys, Ontology

\section{Introduction}

Over the recent years, the number of RDF data sources available on the LOD (Linked Open Data) cloud has led to an explosive growth of the global data space (more than $31 \times 10^{9}$ RDF triples as of September $2011^{1}$ ). In this data space, establishing semantic links between data items can be really useful, since it allows crawlers, browsers and applications to combine information from different sources. These links can be set manually. However, considering the large amount of data available in the Web, some approaches propose methods that generate these links between RDF data sources automatically. Among the different kinds of semantic links that can be established, same-as links express that different identifiers refer to the same world entity (e.g. the same restaurant, the same gene, the same person).

There are a lot of approaches that aim to detect identity links between data items (see [5],[4] or [30] for a survey). In knowledge based approaches the experts declare knowledge that is used to infer identity links between data items. Some of these approaches use rules

Email addresses: pernelle@lri.fr (Nathalie Pernelle), sais@lri.fr (Fatiha Saïs), symeonidou@lri.fr (Danai Symeonidou)

${ }^{1}$ http://www4.wiwiss.fu-berlin.de/lodcloud/state/ that specify conditions that two data items must fulfill in order to be linked. In $[11,28,1]$ these rules are manually defined. In $[10,15,16]$ linkage rules are learnt using genetic programming techniques. $[10,15]$ need a set of reference links to learn these rules while [16] is unsupervised and exploits assumptions on datasets and similarity functions. In [14], the authors use mathematical characteristics of metric spaces to estimate the similarity between instances and filter out instance pairs.

Other approaches such as $[22,8]$ exploit the semantics of the ontology such as keys, functionality of properties and cardinality restrictions. Indeed, these approaches give higher importance to combinations of properties that represent keys or declared as (inverse) functional during the data linking process. In LN2R data linking approach [22] a set of declared keys is exploited by a logical method to generate a set of logical inference rules and by a numerical method to generate a set of similarity functions. The approach ObjectCoref [8] exploits the semantic knowledge like sameAs, (inverse) functional properties and cardinalities to build a seed set of reference links. These links are used to learn discriminative property-value pairs.

Nevertheless, when the ontology represents many concepts and data are numerous, the linking rules or the keys that are needed for the linking step are not often 
available and cannot easily be specified by a human expert. Therefore, we need methods that discover them automatically from the data. Moreover, to the best of our knowledge, the approaches that focus on key discovery $[2,25]$ or learning linking rules $[16,10,15]$ in the semantic Web community use either labeled data to learn the rules [10, 15] or assume that different URIs refer to different world entities (Unique Name Assumption -UNA) [16, 25]. If we consider the overall Linked Open Data cloud (LOD), the UNA is obviously not satisfied, since we can find two different URIs that refer to the same entity. However, it is not uncommon that some datasets considered separately fulfill the UNA. It can be assumed at least for all the datasets generated from relational databases and those created in a way to avoid duplicates like [26]. Recently, W3C has announced the recommendation $\mathrm{R}_{2} \mathrm{RML}^{2}$ as a language for expressing customized mappings from relational databases to RDF datasets. R2RML allows to use primary keys to generate distinct URIs. This will lead to build datasets where the UNA is fulfilled.

When data are heterogeneous, the key discovery problem becomes much more complex. Hence, syntactic variations or errors in literal values may lead to miss keys or to discover erroneous ones. Furthermore, in the semantic Web context, RDF data may be incomplete and asserting the Closed World Assumption (CWA), i.e. what is not currently known to be true is false, as it is proposed in [2], may not be meaningful. Hence, discovering keys on incomplete information needs the use of heuristics to interpret the absence of information.

In this paper, we present an extension of KD2R [27], an automatic approach for key discovery in RDF data sources that conform to OWL ontologies. We aim to discover keys that are composed of several properties. Indeed, non composite keys (e.g. ISBN for books or SSN for persons) are rare in real data. Furthermore, we focus on the discovery of keys that are valid against the considered data. Unlike [2], in this work we do not aim to discover pseudo-keys, that are properties for which some instances are allowed to have the same values.

Like [2, 25], KD2R discovers keys from datasets where the UNA is fulfilled in each of its data sources. As for the Open World Assumption (OWA), KD2R may use either a pessimistic or an optimistic heuristic to interpret the absence of information. In [27], we have defined a first algorithm to compute keys when both heuristics can be applied. In this paper we propose a scalable algorithm which is based on the use of an optimistic heuristic.

\footnotetext{
2http://www.w3.org/TR/r2rml/
}

Unlike the approach that we have presented in [27], in case of different data sources that conform to distinct ontologies we use ontology alignment tools that create mappings between the ontology elements (see [19] for a recent survey on ontology alignment). These mappings will be used to obtain keys that are valid in all the data sources.

To avoid scanning all the data, KD2R discovers first maximal non keys (i.e., a set of properties having the same values for several instances) before inferring the keys. In addition to this, KD2R exploits key inheritance between classes in order to prune the non key search space.

The approach has been implemented and evaluated on five different datasets. To evaluate the quality of the discovered keys, they have been used in a linking process on benchmark datasets. The results that are obtained by LN2R using KD2R keys showed that the use of keys has led to infer more relevant identity links than the ones found when keys were not used. Furthermore, KD2R has been applied on DBpedia data and it has shown that it can scale to millions of triples.

The remainder of this paper is organized as follows. We first describe the data and the ontology model in Section 2 and formalize the problem in Section 3. We present the KD2R approach in Section 4 and the key discovery algorithms in Section 5. Then, in section 6, we present the optimistic algorithm of key discovery. After that, we present the experiment results in Section 7. We conclude our presentation with an overview of related work (Section 8) and concluding remarks (Section 9).

\section{Ontology and Data Model}

We consider $\mathrm{RDF}^{3}$ data sources, each conforming to an $\mathrm{OWL}^{4}$ ontology. The Web Ontology Language (OWL) allows to declare classes and (data or object) properties which can be organized in a hierarchy using the subsumption relation. A set of constraints can also be declared in the ontology. In Figure 1, we present a part of DBpedia ontology concerning restaurants (name space $d b^{5}$ ). The class $d b$ :Restaurant is described by its name, its telephone number, its address and finally the city and the country where it is located. The class $d b$ :Restaurant is a subclass of the class $d b$ :Building.

OWL2 allows us to express keys for a given class: a key hasKey $\left(C E\left(\right.\right.$ ope $_{1}, \ldots$, ope $\left.\left._{m}\right)\left(d p e_{1}, \ldots, d p e_{n}\right)\right)$

\footnotetext{
3 wWw.w3.org/RDF

${ }^{4}$ http: //www.w3.org/TR/owl2-overview

${ }^{5}$ http: //dbpedia.org/ontology/
} 


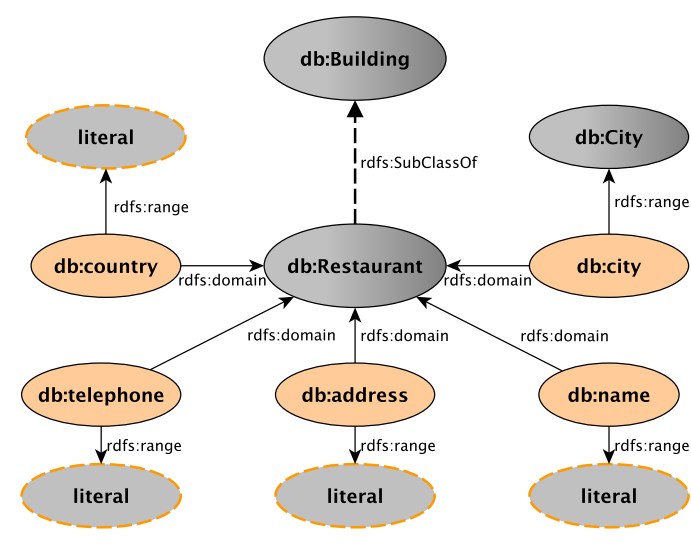

Figure 1: A small part of DBpedia ontology for the restaurants

states that each instance of the class expression $C E^{6}$ is uniquely identified by the object property expressions $o p e_{i}$ and the data property expressions $d p e_{j}$. This means that there is no couple of distinct instances of $C E$ that shares values for all the object property expressions $o p e_{i}$ and all the data property expressions $d p e_{j}$. An ObjectProperty Expression is either an ObjectProperty or Inverse ObjectProperty. The only allowed data property expression is a dataTypeProperty.

For example, we can express that the property expression $\{d b$ :address $\}$ is a key for the class $d b$ :Restaurant using hasKey(db:Restaurant $(()(d b: a d d r e s s))$.

An RDF data source contains a set of class instances described by a set of class facts and property facts. Henceforth, we will use the relational notation: $C(X)$ is used to express that $X$ is an instance of $C$ and $p(X, Y)$ expresses that the couple $(X, Y)$ is an instance of $p$.

We assume that OWL entailment rules [18] are applied on the RDF facts.

For example, the RDF source $s 1$ contains the RDF descriptions of four $d b$ :Restaurant instances.

\footnotetext{
${ }^{6} \mathrm{We}$ consider only the class expressions that represent OWL classes
}

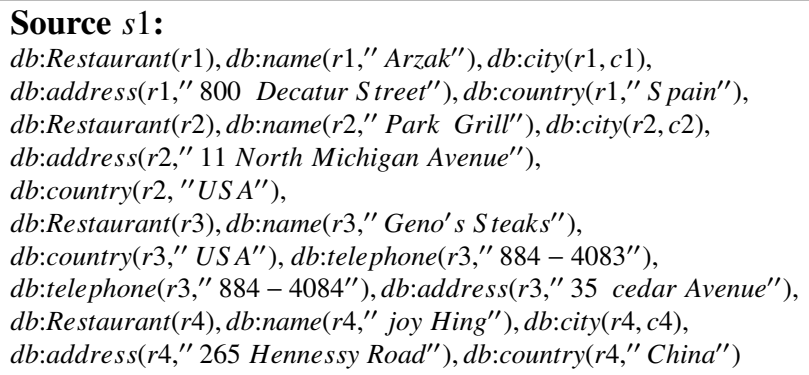

\section{Problem Statement}

Keys express combinations of properties that uniquely identify each instance. For this reason, keys are commonly used in data linking approaches $[22,17,28]$ to infer identity links between instances. The keys are rarely available and not obvious to be declared by a human expert. In this paper, we focus on the automatic discovery of composite keys from data sources where information can be incomplete. We are interested in discovering keys that are valid in several data sources. A key is considered as valid in a data source if, for all pairs of distinct instances, there exists at least a value of a property expression belonging to the key that is different. However, when the UNA is not fulfilled, we cannot be sure if two instances are distinct or not. Hence, it is not obvious to distinguish the following two cases: (i) redundant property values describing data items that refer to the same real world entity and (ii) redundant property values describing data items that refer to two distinct real world entities, i.e. these values instantiate a property expression(s) that is (are) not a key.

Example: Consider an additional instance $d b$ :Restaurant $(r 5)$, in the source $s 1$, with the same value for the property $d b$ :name $\left(r 5,{ }^{\prime \prime}\right.$ Geno's Steaks") as $r 3$. If the UNA is not fulfilled, the probability for the property db:name to be a key will depend on the probability of $r 3$ and $r 5$ to refer to the same restaurant.

Since we are interested in the discovery of valid keys, we only consider data sources where the UNA is fulfilled.

The data sources may not be described using the same ontology. This is why we assume equivalence mappings between classes and properties that are declared or computed by an ontology alignment tool. If we consider that all the data sources are united in a single data source (under an integrated ontology), the UNA would be no longer guaranteed. Therefore, we tackle the problem 
where the keys are first discovered in each data source and then merged according to the given mapping set.

Let $s_{1}$ and $s_{2}$ be two RDF data sources that conform to two OWL ontologies $o_{1}, o_{2}$ respectively.

We consider in each data source $s_{i}$ the set of instantiated property expressions $\mathcal{P} e_{i}=\left\{p e_{i 1}, p e_{i 2}, \ldots, p e_{i N}\right\}$. Let $C_{i}=\left\{c_{i 1}, c_{i 2}, \ldots, c_{i L}\right\}$ be set of classes of the the ontology $o_{i}$. Let $\mathcal{M}$ be the set of equivalence mappings between the elements (property expressions or classes) of the ontologies $o_{1}$ and $o_{2}$. Let $\mathcal{P} e_{1 c}$ (resp. $\mathcal{P} e_{2 c}$ ) be the set of properties of $\mathcal{P} e_{1}$ (resp. of $\mathcal{P} e_{2}$ ) such that there exists an equivalence mapping with a property of $\mathcal{P}_{e_{2}}$ (resp. of $\mathcal{P} e_{1}$ ).

The problem of key discovery that we address in this work is defined as follows:

1. for each data source $s_{i}$ and each class $c_{i j} \in C_{i}$ of the ontology $o_{i}$, such that it exists a mapping between a class $c_{i j}$ and a class $c_{k s}$ of the other ontology $o_{k}$, discover the parts of $\mathcal{P} e_{i}$ that are keys in the data source $s_{i}$

2. find all the parts of $\mathcal{P}_{e_{i c}}$ that are keys for equivalent classes in the two data sources $s_{1}$ and $s_{2}$ with respect to the property mappings in $\mathcal{M}$.

\section{KD2R: Key Discovery approach for Data Linking}

Given two RDF data sources and two domain ontologies, KD2R aims at finding automatically keys for each instantiated class of each ontology of each considered data source. The obtained keys are then merged in order to find keys that are valid in all the considered data sources.

In this section, we will introduce some preliminary definitions and then we will present an overview of KD2R approach.

\subsection{Keys, Non Keys and Undetermined Keys}

We consider that a set of property expressions is a key (c.f. definition 1) for a class if for all pairs of distinct instances of this class, there exists a property expression in this set such that all the values are distinct (objects or literal values). We consider that a set of property expressions is a non key (c.f. definition 2) for a class if there exist two distinct instances of this class that share the same values for all the property expressions of this set.

Since real RDF data sources might contain descriptions that are incomplete, some combinations of property expressions are neither keys nor non keys. More precisely, a set of property expressions is called an undetermined key (c.f. definition 3 ) for a class if it is not a non key and there exist two instances of the class such that the instances share the same values for a subset of the property expressions. The remaining property expressions are unknown for at least one of the two instances.

Distinguishing undetermined keys from keys and non keys allows us to use them in different ways. Using a pessimistic heuristic, the property for which no value is given can take all the values that appear in the data source. Therefore, the undetermined keys will not be considered as keys. Using an optimistic heuristic, the not given property values are different from all the values that appear in the data source for this property. This leads to consider the undetermined keys as keys. These undetermined keys can be validated by a human expert. Indeed, it allows a system to propose to the expert all the candidate keys that can be valid regarding to the dataset(s).

Let $s_{i}$ be an RDF data source that conforms to an OWL ontology $o_{i}$ and is under the UNA.

Definition 1. - Key. A set of property expressions $k_{s_{i} . c}=\left\{p e_{1}, \ldots, p e_{n}\right\}$ is a key for the class $c$ in $s_{i}$ if:

$$
\begin{gathered}
\forall X \forall Y((X \neq Y) \wedge c(X) \wedge c(Y)) \Rightarrow \\
\exists p e_{j}\left(\exists U \exists V p e_{j}(X, U) \wedge p e_{j}(Y, V)\right) \wedge \\
\left(\forall Z \forall W \neg\left(p e_{j}(X, Z) \wedge p e_{j}(Y, W) \wedge(Z=W)\right)\right)
\end{gathered}
$$

We denote $K_{s_{i} . c}$ the set of keys of the class $c$ w.r.t the data source $s_{i}$.

Example. $\quad\{\mathrm{db}$ :address $\} \in K_{\text {s1.db:Restaurant }}$ since the addresses of all the restaurants appearing in the data source $\mathrm{s} 1$ are distinct.

A key $k_{s_{i} . c}$ is minimal if it does not exist a key $k_{s_{i} . c}^{\prime}$ such that $k_{s_{i} . c}^{\prime} \subset k_{s_{i} . c}$.

Definition 2. - Non key. A set of property expressions $n k_{s_{i} . c}=\left\{p e_{1}, \ldots, p e_{n}\right\}$ is a non key for the class $c$ in one data source $s_{i}$ if:

$$
\begin{gathered}
\exists X \exists Y \exists Z_{1}, \ldots, \exists Z_{n}\left(p e_{1}\left(X, Z_{1}\right) \wedge p e_{1}\left(Y, Z_{1}\right) \wedge \ldots \wedge\right. \\
\left.p e_{n}\left(X, Z_{n}\right) \wedge p e_{n}\left(Y, Z_{n}\right) \wedge(X \neq Y) \wedge c(X) \wedge c(Y)\right)
\end{gathered}
$$

We denote $N K_{s_{i} . c}$ the set of non keys of the class $c$ w.r.t the data source $s_{i}$.

Example. $\{\mathrm{db}$ :country $\} \in N K_{s 1 \text {.Restaurant }}$ since there are two restaurants that are located in the same country (USA) in the data source $s 1$. 
(a) KeyFinder for one data source

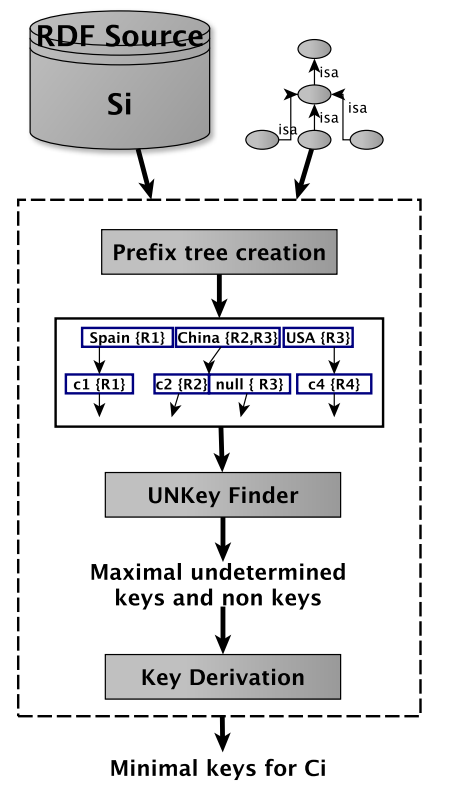

(b) Key merge for two data sources

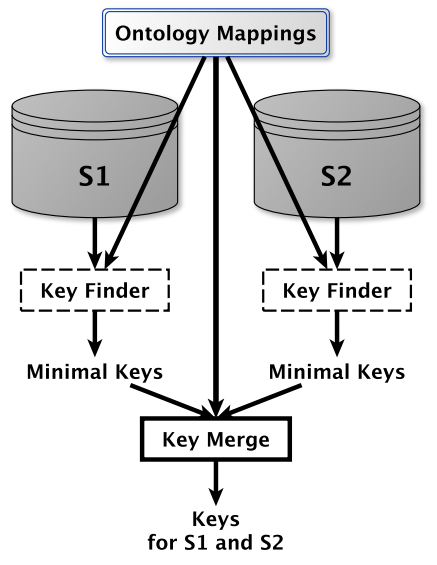

Figure 2: Key Discovery for two data sources

A non key $n k_{s_{i} . c}$ is maximal if it does not exist a non key $n k_{s . c}^{\prime}$ such that $n k_{s . c} \subset n k_{s . c}^{\prime}$.

Definition 3. - Undetermined Key. A set of property expressions $u k_{s_{i} . c}=\left\{p e_{1}, \ldots, p e_{n}\right\}$ is an undetermined key for the class $c$ in $s_{i}$ if:

- (i) $u k_{s_{i} . c} \notin N K_{s_{i} .}$ and

- $(i i) \exists X \exists Y\left(c(X) \wedge c(Y) \wedge(X \neq Y) \wedge \forall p e_{j}\right.$

$$
\left(\left(\exists Z\left(p e_{j}(X, Z) \wedge p e_{j}(Y, Z)\right) \vee\right.\right.
$$

$$
\left.\left.\nexists W\left(p e_{j}(X, W) \vee \nexists W p e_{j}(Y, W)\right)\right)\right)
$$

We denote $U K_{s_{i} . c}$ the set of undetermined keys of the class $c$ w.r.t the data source $s$.

Example. $\{$ db:country, db:city $\} \in U K_{\text {s1. Restaurant }}$ since it is not a non key and there are two restaurants in the same country(USA) but one of them doesn't contain any information about the city where it is located.

An undetermined key $u k_{s_{i} . c}$ is maximal if it does not exist an undetermined key $u k_{s_{i} . c}^{\prime}$ such that $u k_{s_{i} . c} \subset u k_{s_{i} . c}^{\prime}$.

\subsection{KD2R overview}

The most naive automatic way to discover the keys is to check all the possible combinations of property expressions that refer to a class. Assume that we have a class that is described by 15 properties. In this case, the number of candidate keys is $2^{15}-1$. In order to minimize the number of computations, we propose a method inspired by [23] which first retrieves the set of maximal non keys (i.e. combinations of property expressions that share the same values for at least two instances) and then computes the set of minimal keys, based on the set of the discovered non keys. Indeed, to make sure that a set of property expressions is a key, we have to scan the whole set of instances of a given class. On the other hand, finding two instances that share the same values for the considered set of property expressions would suffice to be sure that this set is a non key.

In Figure 2 we show the main steps of the KD2R approach. Our method discovers the keys for each RDF data source independently. In each data source, KD2R is applied on the classes in topologically sorted order. In this way, the keys that are discovered in the superclasses are exploited in the processing of their subclasses. For a given data source $s_{i}$ and a given class $c$ we apply KeyFinder (Algorithm 1) which aims at finding keys for the class $c$ that are valid in the data source $s_{i}$. 
KeyFinder starts by building a prefix tree for this class to represent its instances (see Figure 2(a)). Using this representation the sets of maximal undetermined keys and maximal non keys are computed. These sets of undetermined keys and non keys, are used to derive the set of minimal keys. The obtained keys are then merged in order to compute the set of keys that are valid for both data sources (see Figure 2(b)).

\section{KD2R general algorithm}

The main algorithm of the $\mathrm{KD} 2 \mathrm{R}$ approach is KeyFinder (Algorithm 1), which retrieves for each RDF data source, conforming to an OWL ontology, the minimal keys that can be added to the classes of the ontology. KeyFinder, starts by computing the topological order of the classes by exploiting the subsumption relation between them.

For each class, KeyFinder builds an intermediate prefix tree (see Algorithm 7) which is a compact representation of the class instances in the data source. Then the final prefix tree (see Algorithm 3) is generated in order to take into account the possible unknown property values. Using the final prefix tree the UNKFinder method is called to retrieve the maximal non keys and the maximal undetermined keys using inherited keys if there exist. Finally, KeyFinder computes the complete set of minimal keys for each class.

KeyFinder (Algorithm 1) corresponds to the pessimistic heuristic. To consider the optimistic one, it suffices to call the keyDerivation (6), method using only the set of non keys $N K_{s . c}$.

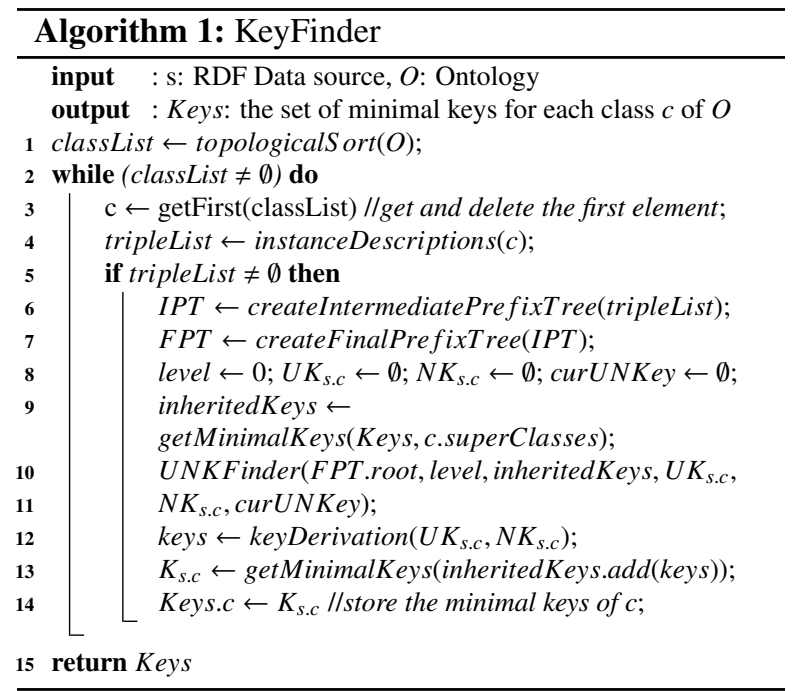

\subsection{Prefix Tree creation}

We now describe the creation of the prefix tree which represents the instances of a given class in one data source. We consider that the RDF descriptions of the instances are saturated using the OWL entailment rules [18].

Each level of the prefix tree corresponds to a property expression pe. Each node contains a set of cells and a variable number of cells. Each cell contains:

1. a cell value: (i) when pe is a property, the cell value is one literal value, one URI instantiating its range or a null value and (ii) in case pe is a inverse property, the cell value is one URI instantiating its domain or an artificial null value.

2. a URI list (UL): (i) when pe is a property the URI list is the set of URIs instantiating its domain and having as range the cell value, and (ii) in case $p e$ is an inverse property, the URI list is the set of URIs instantiating its range and having as domain the cell value.

3. a URI list (NUL): the list of URIs for which the property expression value is unknown and for which we have assigned the cell value (null or not).

4. a pointer to a single child node.

Each prefix path corresponds to the set of instance URIs that share the cell values for all the property expressions involved in the path.

In order to consider the cases where property values are not given in the data source, we create first an intermediate prefix tree. In this intermediate prefix tree, an artificial null value is created for those properties. The final prefix tree is generated by assigning all the existing cell values of one node to the cell that contains the artificial null value.

\subsubsection{Intermediate Prefix Tree creation}

In order to create the intermediate prefix tree we use the set of all property expressions that appear at least in one instance description of the considered class. For each value of a property expression, if there is no existing cell value with the same value a new cell is created and the URI list UL is initialized with the instance URI. When a property expression does not appear in the description of an instance, we create or update, in the same way, a cell with an artificial null value. This intermediate prefix tree creation is done by scanning the data only once. 


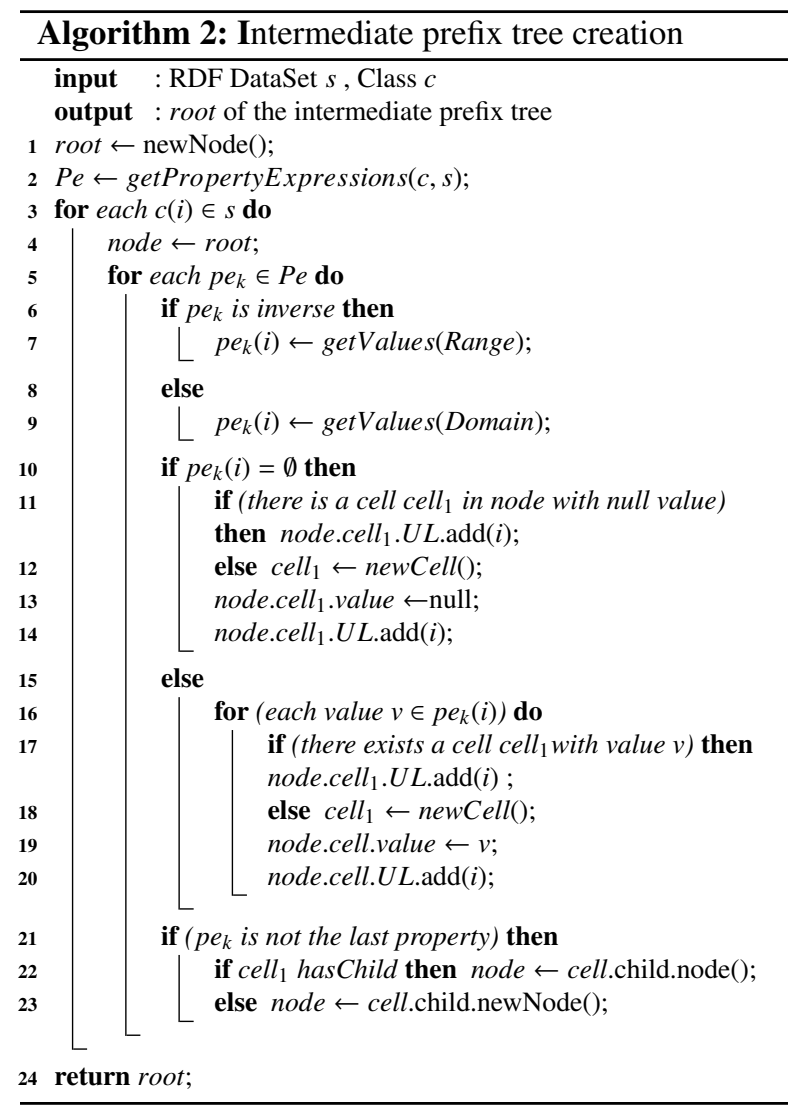

Example of Intermediate Prefix Tree creation. The creation of the intermediate prefix tree (see Figure 3) starts with the first entity which is the db:Restaurant $r 1$. A new cell is created in the root node describing the name of the country in which the restaurant is located. The next information concerning this restaurant is the city where it is located. To store this information a new node will be created as a child node of the cell "Spain". A new cell is created in this node to store the value $c 1$. The process continues until all the information about an entity are represented in the tree. For each new entity, the insertion begins again from the root.

In figure 3, we give the intermediate prefix tree for the class $d b$ : Restaurant instances of the RDF data source $s 1$ described in section 2 .

\subsubsection{Final Prefix Tree creation}

We generate a final prefix tree from the intermediate prefix tree (see Algorithm 3) by assigning the set of the possible values contained in the cells of one node to the artificial null value of this node, if it exists. We use the URI list NUL to store the URIs for which the property expression value was unknown. This information will be used by UNKFinder (Algorithm 5) to distinguish non keys from undetermined keys.

Example of Final Prefix Tree creation. As we can see in Figure 3 there are two restaurants in USA: $r 2$ and $r 3$. The restaurant $r 2$ is located in $c 2$ while there is no information about the location of $r 2$. This absence is represented by a null cell in the intermediate prefix tree (see Figure 3). Therefore, we assign the value $c 2$ for the property $d b$ : city of $r 3$. The URI list NUL is now $\{r 2, r 3\}$ and $r 3$ is stored in the list NUL (see Figure 5(b)). This assignation is performed using the mergeCells function. This process will be applied recursively to the children of this node (see Figure 5(c)) in order to: (i) merge the cells of the child nodes that contain the same value and (ii) to replace the null values by the possible ones. In figure 4 , we give the final prefix tree of the RDF data described in section 2.
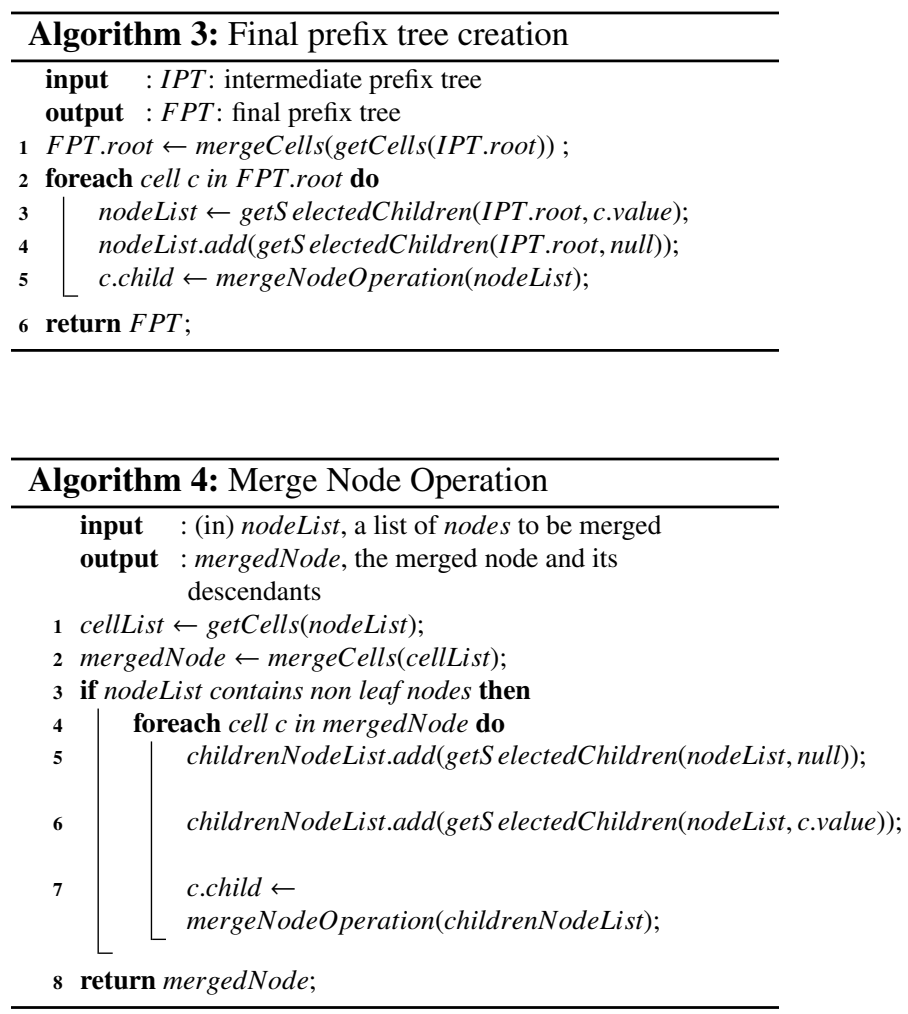

\subsection{Undetermined and non key discovery (UNKFinder)}

UNKFinder algorithm aims at retrieving the maximal undetermined keys $U K_{s . c}$ and the maximal non keys $N K_{\text {s.c }}$ from a final prefix tree(see Algorithm 3). The 


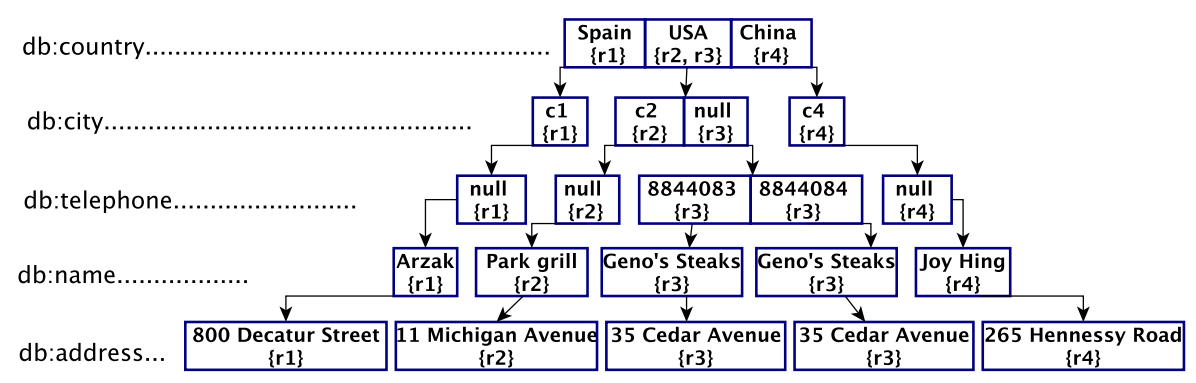

Figure 3: Intermediate prefix tree for the $d b$ : Restaurant class instances

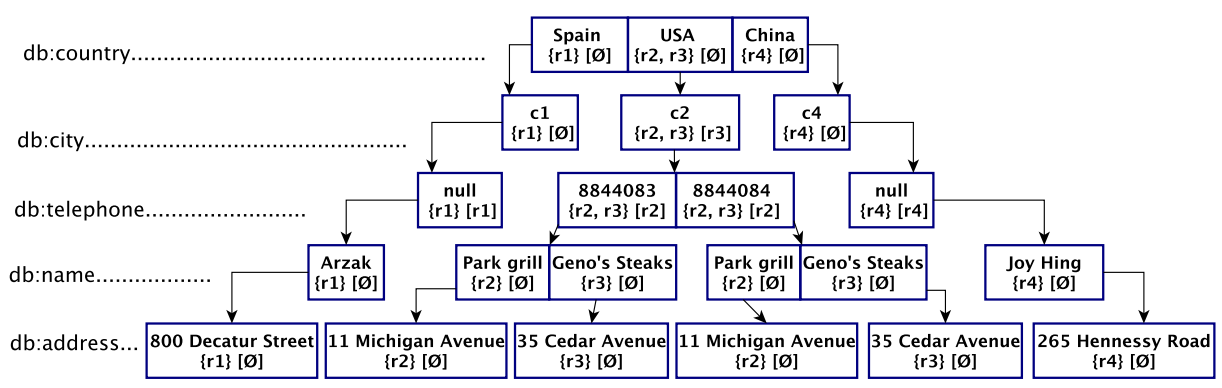

Figure 4: Final prefix tree for the db:Restaurant class instances

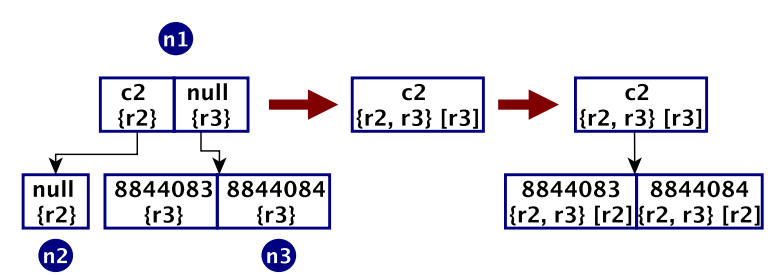

a)

b)

c)

traversal of the prefix tree is depth-first. This method searches the biggest combination of property expressions having values that are shared by more than one instance in the dataset, using a depth-first traversal of the tree. This means that this combination of property expressions represents either a non key or an undetermined key.

More precisely, when a leaf node is reached, if one of the cells of this leaf node contains a list of URIs (UL) with size $>1$, we are sure that the constructed list of property expressions (curUNKey) is either a non key

or an undetermined key. If one of the URIs of UL is obtained by a merge operation with a null value then curUNKey is an undetermined key otherwise it is a non key.

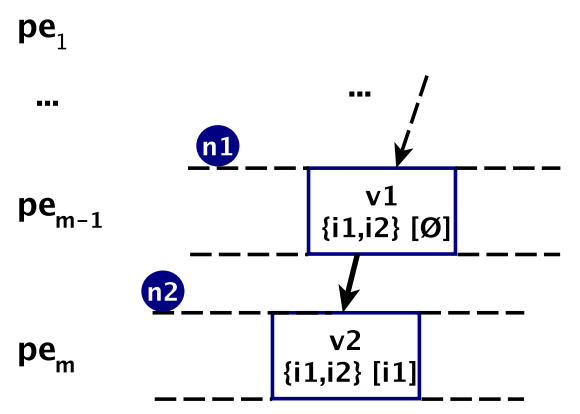

Figure 6: Example 1

In the example of Figure 6, the combination of property expressions $\left\{p e_{1}, \ldots, p e_{m}\right\}$ is an undetermined key.

In addition to this, when the size of the union of all the URI lists UL of the leaf node is greater than 1, we 
know that curUNKey that is constructed before adding the leaf level is a non key or an undetermined key (same criteria than above to distinguish them).

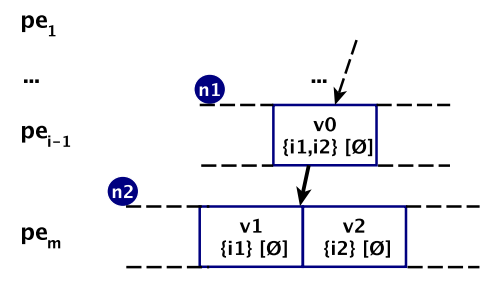

Figure 7: Example 2

In the example of Figure 7 , in the node $n_{2}$, since $\left|\left\{i_{1}\right\} \cup\left\{i_{2}\right\}\right|>1$, then $\left\{p e_{1}, \ldots, p e_{m-1}\right\}$ is a non key or an undetermined key.

In order to generate all the possible combinations of property expressions, we need to ignore some of them (i.e., level(s) in the prefix tree). Therefore the descendants of the ignored level(s) have to be merged using the merge node operation (see Algorithm 4).

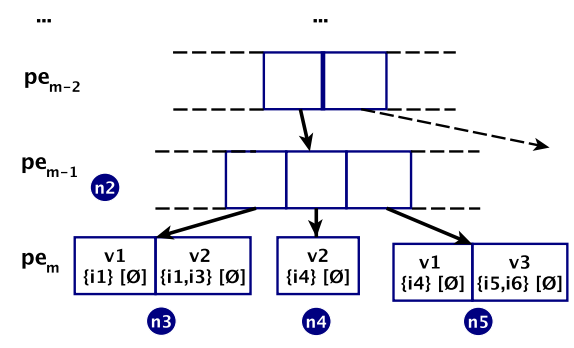

Figure 8: Example 3

In the example of Figure 8 we illustrate how the merge node operation is used to build all the possible prefix trees corresponding to the possible combinations of property expressions. The first list of property expressions $\left\{p e_{1}, \ldots, p e_{m-1}, p e_{m}\right\}$ is tested successively on the leaf nodes $n_{3}, n_{4}$ and $n_{5}$.

Then, $p e_{m-1}$ is suppressed from this combination thanks to the merge node operation applied on the children of $n_{2}$. The new prefix tree is shown bellow in Figure 9 , where $n_{6}$ represents the result of the merge operation on $n_{3}, n_{4}$ and $n_{5}$. This operation is reapplied recursively on the new prefix trees obtained from the merge.

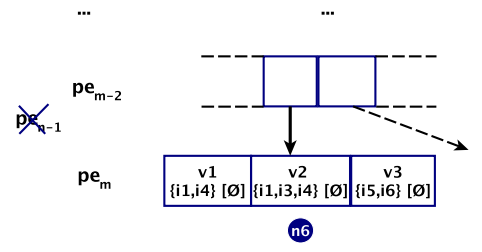

Figure 9: Example 4

To ensure the scalability of the undetermined and non key discovery, UNKFinder performs three kinds of pruning:

(A) The subsumption relation between classes is exploited to prune the prefix tree traversal. Indeed, when a key is already discovered for a class using one data source, then this key is also valid for all the subclasses in this data source. Thus, parts of the prefix tree are not explored.

Example: let $k_{s . c 1}=\left\{\left\{p e_{1}, p e_{3}\right\},\left\{p e_{2}, p e_{4}\right\}\right\}$ be the set of keys of $c_{1}$. Let $c_{2}$ be a subclass of $c_{1}$ in the ontology. Let consider the prefix tree for $c_{2}$ showed in Figure 10.

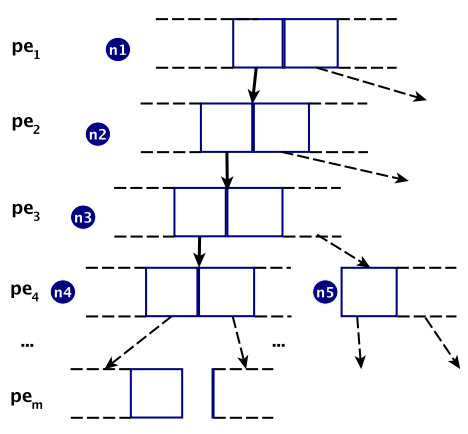

Figure 10: Example 5

When curUNKey $=\left\{p e_{1}, p e_{2}, p e_{3}\right\}$ the pruning is applied because curUNKey includes one of the keys of $c_{1}$ (i.e., $\left\{p e_{1}, p e_{3}\right\}$ ). Therefore, the subtree rooted at $n_{3}$ is not explored.

(B) When all the further new combinations of property expressions in a given path cannot lead to new 


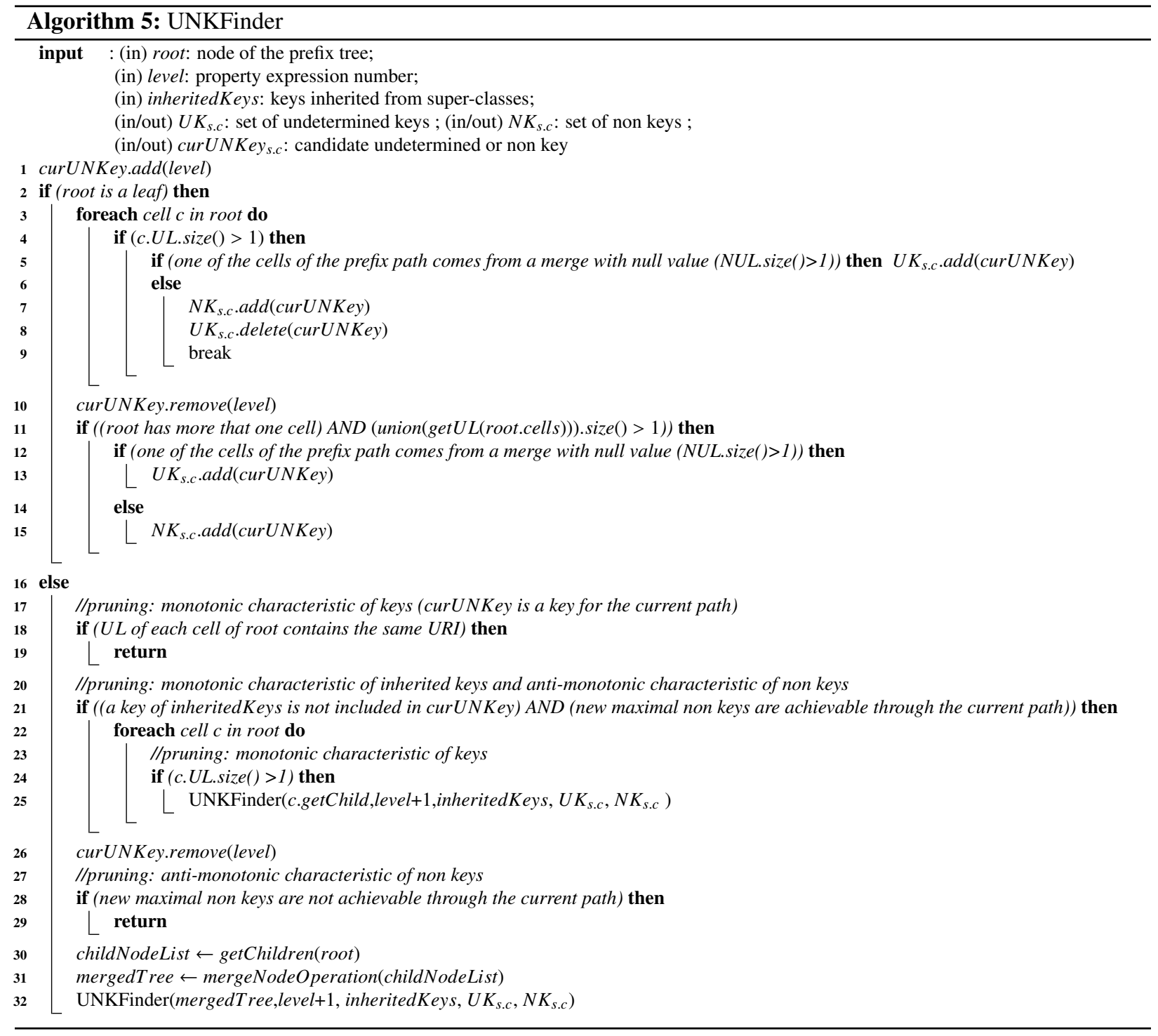


maximal non keys then the exploration of this path stops.

Example: let $N K_{s . c}=\left\{\left\{p e_{1}, p e_{2}, p e_{3}\right\}\right\}$ be the set of already discovered non keys. Suppose that curNKey $=\left\{p e_{1}\right\}$. If the remaining levels of the prefix tree do only correspond to the property expressions $p e_{2}$ and/or $p e_{3}$ then the children of the current node are not explored.

(C) The monotonic characteristic of keys, i.e. if $\{A B\}$ is a key then all the supersets of $\{A B\}$ are also keys. Thus, when a node describes only one instance we are sure that adding more property expressions in the current path will not lead to a non key.

For instance, on the RDF data source $s 1$ described in section 2, we obtain the following sets of maximal undetermined keys and maximal non keys, for the class $d b:$ Restaurant:

$U K_{s 1 . d b: \text { Restaurant }}=\{\{$ db:telephone, db:city, db:country $\}\}$

$N K_{s 1 . d b: \text { Restaurant }}=\{\{d b:$ country $\}\}$

\subsubsection{Key derivation}

Once the sets of maximal undetermined keys and maximal non keys are discovered from a given data source for one class, we derive the set of minimal keys. The main idea is that a key is a set of property expressions that is not included or is not equal to any maximal non key or undetermined key. Thus, to build all these sets of property expressions, for each maximal non key and undetermined key, we retain the property expressions that do not belong to this non key or undetermined key. Then, the obtained property expressions are combined using a cartesian product and only the minimal sets are kept.

More precisely, to derive the minimal keys $K_{s . c}$, we first compute the union of $N K_{\text {s.c }}$ and $U K_{\text {s.c }}$ and select the maximal sets of property expressions (see Algorithm 6). For each selected set of property expressions, we compute the complement set with respect to the whole set of instantiated property expressions. Then we apply the cartesian product on the obtained complement sets. Finally, we remove the non-minimal keys $k_{s . c}$ from the obtained multi-set $K_{s . c}$.

Example. In the $\mathrm{db}$ :Restaurant example we have:

$U K_{s 1 . d b: \text { Restaurant }}=\{\{d b$ :telephone,

$d b:$ city, db:country\}\} and

$N K_{s 1 . d b: \text { Restaurant }}=\{\{d b:$ country $\}\}$.

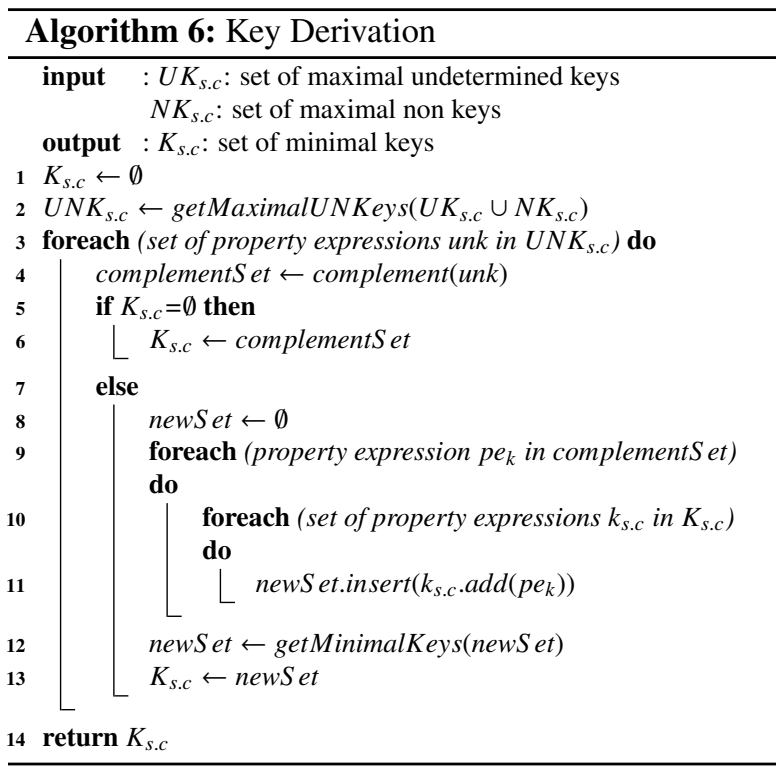

The set of maximal set of property expressions is: $\{\{d b$ :telephone, $d b$ :city, db:country $\}$.

Its complement set is:

$\{d b$ :address $\},\{d b:$ name $\}$.

Since there is only one set of property expressions, we obtain: $K_{s 1 . d b: \text { Restaurant }}=\{\{d b: a d d r e s s\},\{d b:$ name $\}$.

\subsubsection{Multi-source Keys}

When keys are discovered from two data sources that conform to two different ontologies, we compute the keys that are valid in both data sources. The keys are expressed using the common vocabulary. First, for each data source and class we delete from $K_{s . c}$ all the keys that contain property expressions that do not belong to $\mathcal{P} e_{i c}$ (i.e., the set of mapped properties). Then, for each pair of equivalent classes we compute the cartesian product between their set of minimal keys. Finally, we select only the minimal ones. This way we guarantee that the obtained keys are valid in both data sources.

For example, consider two data sources $D=\{s 1, s 2\}$,

if $K_{s 1 . d b: \text { Restaurant }}=\{\{d b:$ address $\}$,

$\{d b:$ name $\}\}$ and

$K_{s 2 . d b: \text { Restaurant }}=\{\{d b:$ telephone,

$d b:$ city $\},\{d b:$ name $\}\}$

then the multi-source keys will be:

$K_{D: \text { Restaurant }}=\{\{d b$ : telephone, $d b:$ address, $d b$ : city\}, $\{d b:$ name $\}$. 


\section{Optimistic Algorithm for Key Discovery}

When the optimistic approach is applied a more efficient key discovery method can be performed. Indeed, considering that each null value can be one of the already existing ones, means that we have to assign all the values to each not given one. This makes the pessimistic approach not scalable when the data are very incomplete. As we have already mentioned the pessimistic approach considers that missing values can be any of the already existing values appearing in the data. Using this approach many keys can be lost due to data incompleteness. For example, if we consider a dataset describing 1000 people. One of the properties is mobilePhone and it is given for 999 people. Even if all the 999 values of mobilePhone are pairwise distinct for each person, mobilePhone will not be discovered using the pessimistic approach. Nevertheless, mobilePhone will be discovered as key using the optimistic approach.

The keys that are discovered by the optimistic approach correspond to the union of the keys (see Definition 1) and the undetermined keys (see Definition 3). The optimistic keys are defined in Definition 4, as follows.

Definition 4. - Optimistic Key. A set of property expressions $k_{s_{i} . c}=\left\{p e_{1}, \ldots, p e_{n}\right\}$ is an optimistic key for the class $c$ in $s_{i}$ if:

$$
\begin{gathered}
\forall X \forall Y((X \neq Y) \wedge c(X) \wedge c(Y)) \Rightarrow \\
\left(\forall Z \forall W \neg\left(p e_{j}(X, Z) \wedge p e_{j}(Y, W) \wedge(Z=W)\right)\right)
\end{gathered}
$$

We denote $K_{s_{i} . c}$ the set of optimistic keys of the class $c$ w.r.t the data source $s_{i}$.

Using the optimistic approach, there is no more need to build the intermediate prefix tree (see Algorithm 2). Indeed, using Algorithm 7, we can build directly the final prefix tree, since no null values have to be merged.

\section{Experiments}

In this section we present the results of the experiments obtained from several datasets. First, we give the obtained keys from each dataset. For some of them we give the obtained results using both the pessimistic and the optimistic approaches. Then, in section 7.2 the scalability of our method (optimistic heuristic) is demonstrated using two datasets extracted from DBpedia that consist of millions of triples. The evaluation of the quality of the discovered keys is performed using a data linking tool and we have measured the

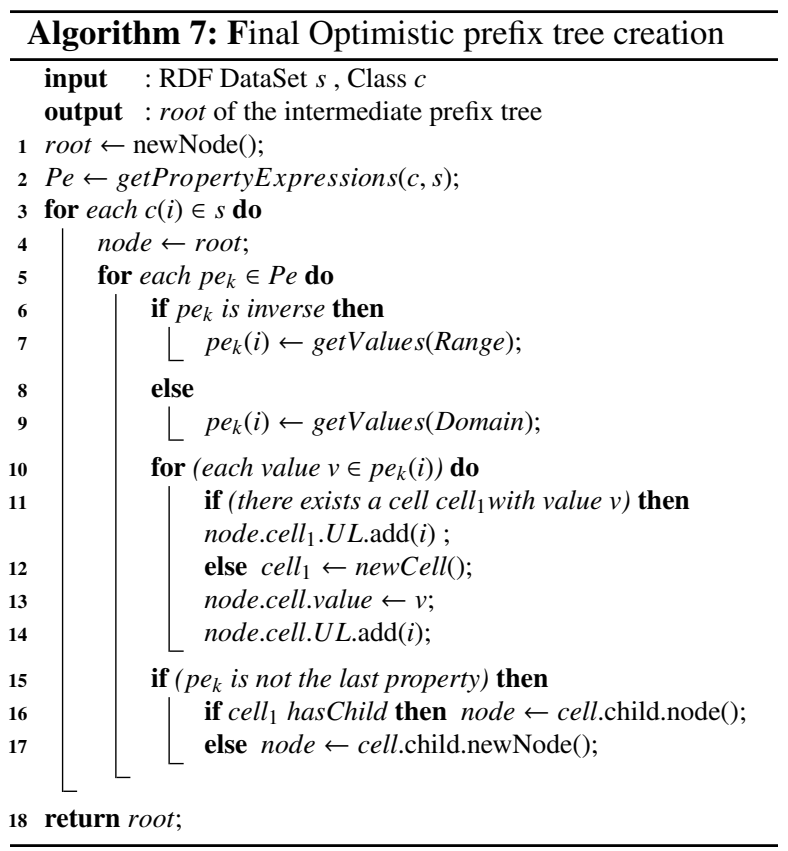

well known recall, precision and F-Measure. The obtained results are presented in two parts: (i) in subsection 7.3.1 we give the obtained recall, precision and FMeasure, on one dataset, when the keys are applied using equality between values (instead of using similarity scores) and when the linking decisions are not propagated (e.g. "same restaurants then, same addresses, then same cities, then, ..."); and (ii) in section 7.3.2 we present the obtained recall, precision and F-measure, on the other datasets, when the keys are applied all together using similarity scores between values and where the linking decisions are propagated thanks to LN2R tool (see section 7.3.2).

\subsection{Evaluation of key discovery}

We have tested KD2R on six RDF datasets ${ }^{7}$. The two first datasets have been used in the OAEI-Ontology Alignment Evaluation Initiative $2010^{8}$, in the Instance Matching track. The three following datasets have been collected from the Web of data. The last dataset has been taken from the OAEI-Ontology Alignment Evaluation Initiative $2012^{9}$. Each dataset contains two RDF data sources and two OWL ontologies. UNA is declared for each RDF data source of the six datasets. For each dataset, we discovered the keys using KD2R. Table 1

\footnotetext{
${ }^{7}$ http://www.lri.fr/ sais/KD2R-Datasets

${ }^{8}$ http://oaei.ontologymatching.org/2010/

${ }^{9}$ http: //oaei.ontologymatching.org/2012/
} 
displays some statistics of the used datasets: the number of triples, the number of instances per class and the number of properties per class.

In the following, we describe each dataset and present the set of keys that are discovered by KD2R.

\subsubsection{KD2R results on OAEI 2010 datasets}

The first dataset $D 1$ consists of 2000 instances of the classes Person and Address (see Table 1). In the Ontology:

- a Person instance is described by the data type properties: givenName, state, surname, dateOfBirth, socS ecurityId, phoneNumber, age and the object property hasAddress.

- an Address instance is described by the data type properties: street, houseNumber, postCode, isInS uburb ${ }^{10}$ and the object property hasAddress.

Both RDF data sources of the dataset $D 1$ contain 500 instances of the class Person and 500 instances of Address.

KD2R has discovered the four following keys for the classes Person and Address in the dataset D1, using the pessimistic heuristic:

$K_{D 1 . \text { Person }}=\{\{$ socS ecurityId $\},\{$ hasAddress $\}\}$

$K_{D 1 . A d d r e s s}=\{\{$ isInS uburb, postcode, houseNumber $\}$, $\{$ inverse(hasAddress) $\}$ \}.

Applying the optimistic heuristic, KD2R has discovered the thirteen following keys for the Person and Address classes in the same dataset:

$K_{D 1 . \text { Person }}=\quad\{\{$ socSecurityId $\}, \quad\{$ hasPhone $\}$ \{hasAddress\}, $\quad$ dateOfBirth, givenName\}, \{dateOfBirth, age $\}$,

$\{$ surname, dateOfBirth $\},\{$ surname, givenName $\}\}$

$K_{D 1 . \text { Address }}=\{\{$ street, houseNumber $\},\{$ street, isInSuburb $\},\{$ houseNumber, isInS uburb $\},\{$ postCode, is InS uburb $\}$ $\{$ street, postCode $\},\{$ inverse(hasAddress) $\}$ \}.

In optimistic heuristic, all the undetermined keys are considered as keys. In the Person dataset D1, there are a lot of not instantiated properties. Thus, we have obtained a lot of undetermined keys. This has led to a set of keys that is bigger than the one obtained using

\footnotetext{
${ }^{10}$ in the ontology of the second data source $i \operatorname{In} S$ uburb is declared as an object property. Since, it was the unique difference between the two ontologies, we have chosen to rewrite the second data source using the first ontology. An analogous processing has been performed on the second dataset.
}

the pessimistic heuristic.

The second dataset $D 2$ describes 1730 instances of Restaurant and Address classes (see Table 1). It corresponds to the first version of the OAEI 2010 restaurant dataset that contains bugs. In the provided ontology we have:

- a Restaurant instance is described using the datatype properties properties name, phoneNumber, hasCategory and the object property hasAddress.

- an Address instance is described using the datatype properties street, city and the object property hasAddress.

The first RDF data source s1 describes 113 Address instances and 113 Restaurant instances. The second RDF data source s 2 describes 752 Restaurant instances and 752 Address instances.

The five keys that are obtained for Restaurant and Address classes in the dataset D2, under the pessimistic heuristic, are as follows:

$K_{D 2 . \text { Restaurant }}=\{\{$ phoneNumber, name $\}$, $\{$ phoneNumber, hasCategory\}, \{name, hasCategory $\},\{$ hasAddress $\}$ \} $K_{D 2 . \text { Address }}=\{\{$ inverse $($ hasAddress $)\}\}$.

Since D2 does not contain any undetermined keys, the obtained results are the same for both the optimistic and pessimistic heuristic (see section 4.1).

\subsubsection{KD2R results on GFT-ChefMoz dataset}

The GFT-ChefMoz dataset is composed of two RDF data sources and two OWL ontologies. The first data source has been extracted from the ChefMoz repository published on the Linked Open Data Cloud (LOD). The , second data source was obtained from Google Fusion tables service [7], by [20]. In order to enforce the UNA in the ChefMoz dataset we used the linking tool LN2R without keys (see Section 7.3.2). The results have been manually validated and the duplicates have been suppressed.

The GFT data source $s 1$, collected from the LOD, contains 1575 instances of the class Restaurant (see 1). In the ontology a restaurant is described by the data type properties: title, address, cuisine, city.

The ChefMoz data source $s 2$ describes 32586 instances of the class Restaurant (see Table 1). In this ontology, restaurants are described using more properties than in ontology of the data source $s 1$. 


\begin{tabular}{|c|c|c|c|c|}
\hline dataset & source & \#triples & \#instances (per class) & \#properties (per class) \\
\hline \multirow[t]{2}{*}{ Person 1 (D1) } & \multirow[t]{2}{*}{ s1 } & \multirow[t]{2}{*}{5801} & Person: 500 & Person: 7 \\
\hline & & & Address: 500 & Address: 6 \\
\hline \multirow[t]{2}{*}{ Person 1 (D1) } & \multirow[t]{2}{*}{ s2 } & \multirow[t]{2}{*}{6230} & Person: 500 & Person: 7 \\
\hline & & & Address: 500 & Address: 6 \\
\hline \multirow[t]{2}{*}{ Restaurant (D2) } & \multirow[t]{2}{*}{ s1 } & \multirow[t]{2}{*}{891} & Restaurant: 113 & Restaurant: 4 \\
\hline & & & Address: 113 & Address: 3 \\
\hline \multirow[t]{2}{*}{ Restaurant (D2) } & \multirow[t]{2}{*}{ s2 } & \multirow[t]{2}{*}{3347} & Restaurant: 752 & Restaurant: 4 \\
\hline & & & Address: 752 & Address: 3 \\
\hline GFT \& ChefMoz (D3) & s1 (GFT) & 4494 & Restaurant: 1349 & Restaurant: 4 \\
\hline GFT \& ChefMoz (D3) & s2 (ChefMoz) & 153300 & Restaurant: 32686 & Restaurant: 4 \\
\hline \multirow[t]{5}{*}{ Film (D6) } & \multirow[t]{5}{*}{ s1 } & \multirow[t]{5}{*}{117076} & Film: 1288 & Film: 8 \\
\hline & & & Creatures: 8401 & Creatures: 9 \\
\hline & & & Location: 2471 & Location: 8 \\
\hline & & & Language: 67 & Language: 4 \\
\hline & & & Budget: 101 & Budget: 2 \\
\hline \multirow[t]{5}{*}{ Film (D6) } & \multirow[t]{5}{*}{$\mathrm{s} 2$} & \multirow[t]{5}{*}{129429} & Film: 1288 & Film: 8 \\
\hline & & & Creatures: 8401 & Creatures: 9 \\
\hline & & & Location: 2471 & Location: 8 \\
\hline & & & Language: 67 & Language: 4 \\
\hline & & & Budget: 101 & Budget: 2 \\
\hline
\end{tabular}

Table 1: Statistics on OAEI 2010 and GFT \& ChefMoz datasets

Equivalence mappings have been declared between the four properties of GFT (s1) and the properties of ChefMoz (s2).

$\mathrm{KD} 2 \mathrm{R}$ has discovered the following keys for the class Restaurant in the data source $\mathrm{s} 1$, using the pessimistic heuristic:

$K_{s 1 . \text { Restaurant }}=\{\{$ address $\},\{$ city, title $\}\}$

The key that is obtained for Restaurant in the data source s2 is the following composite key, using the pessimistic heuristic:

$K_{\text {s2. Restaurant }}=\{\{$ title, address $\}\}$

After the merge, the obtained multi-source key is: $K_{\text {D3.Restaurant }}=\{\{$ title, address $\}\}$

Using the optimistic heuristic, the keys obtained on each data source are different but the key obtained after their merge is equal to the one obtained using pessimistic heuristic.

\subsubsection{KD2R results on DBpedia dataset}

In order to show the scalability of our method, we have applied KD2R on two datasets extracted from DB- pedia $^{11}$ : the first dataset contains descriptions of persons and the second one of natural places (see table 2). One of the characteristics of DBpedia is that the UNA is not fulfilled. All the keys that can be discovered on such a dataset would still remain valid even if the duplicates are removed. However, some of the possible minimal keys can be lost. In the worst case scenario, two duplicates are represented by the same property values. Hence, no keys can be found using these properties. In DBpedia, we have observed that some people are represented several times using distinct URIs, but in different contexts (e.g. one soccer-player is represented using several URIs, but for each URI the description concerns its transfer into an new club). Therefore, in such cases keys can be discovered.

On small data sources such as OAEI data sources or GFT (less than 10000 triples), KD2R can be applied using the pessimistic or the optimistic heuristic. Nevertheless, on large datasets such as DBpedia persons (more than 5.6 millions of triples) or DBpedia natural places (more than 1.6 millions of triples), the pessimistic approach cannot by used. Indeed, such datasets contain a lot of properties that are rarely instantiated which leads to a final prefix tree that contains too many nodes (i.e.

\footnotetext{
${ }^{11}$ http://dbpedia.org/Downloads37
} 
assignation of all the possible values to the artificial "null" values in the prefix tree). Hence, in such cases only the optimistic heuristic can be applied. Moreover, in our experiments we have considered only the properties that are instantiated for at least $T$ distinct Person and NaturalPlace instances.

The first dataset contains 763644 instances of the class Person which corresponds to 5639680 RDF triples. The second dataset contains 49887 instances of the class NaturalPlace, or 1604347 RDF triples. To show how the inherited keys are exploited, KD2R has been applied on the class NaturalPlace, its subclass BodyOfWater and on the class Lake which is a subclass of the class BodyOfWater.

For the class Person of D4, when $T$ is equal to $20 \%$, the set of obtained keys is as follows: $K_{D 4 . P e r s o n}=\{\{$ squadnumber, birthplace $\}$, \{squadnumber, birthdate $\}, \quad\{$ currentmember, birthplace $\},\{$ currentmember, name $\},\{$ squadnumber, name $\},\{$ currentmember, birthdate $\}$ \}

When $T$ is equal to $10 \%, \mathrm{KD} 2 \mathrm{R}$ obtains 17 additionnal composite keys, such as \{name, position, deathdate and name, occupation, birthdate, activeyearstartyear, birthplace \} \}

For the class NaturalPlace of D5, when $T$ is equal to $20 \%$, the set of obtained keys is:

$K_{\text {D5.NaturalPlace }}=\{\{$ name, district, elevation $\}$, \{sourcecountry, location\}, \{country, district, long\}, \{district, sourcecountry, elevation\}, \{sourcecountry, long $\}, \quad\{$ district, location $\}$, \{name, lat, district $\},\{$ country,locatedinarea $\},\{$ lat, district, elevation\}, \{lat, sourcecountry\}, \{location, locatedinarea $\}, \quad\{$ sourcecountry, locatedinarea $\}$, \{district,locatedinarea $\}, \quad\{$ name, district, point $\}$, \{country, lat, district\}, \{name, district, long\}, $\{$ district, elevation, long $\},\{$ country, sourcecountry, elevation $\},\{$ country, district, point $\},\{$ district, point, elevation $\},\{$ sourcecountry, point $\}$ \}

For the 33993 instances of the class BodyOfWater, we have found 13 keys, four of them are subsets of some minimal keys that are inherited from NaturalPlace like $\{$ lat, district $\}$. The other minimal keys belong to the set of minimal keys inherited from NaturalPlace.

For the 9438 instances of the class Lake, we have found 7 minimal keys, three of them are subsets of some minimal keys that are inherited from BodyOf Water like $\{$ sourceCountry\}. The other minimal keys belong to the set of minimal keys inherited from BodyOfWater.

\subsubsection{KD2R results on IIMB dataset}

The last dataset used in the experiments is the IIMB dataset (ISLab Instance Matching Benchmark), D6. This benchmark is used in the instance matching track of the Ontology Alignment Evaluation Initiative (OAEI 2011 \& 2012). An initial dataset that describes movies (films, actors, directors, etc.) was extracted from the web (file 0). The classes contained in this dataset except for movies, are creatures, languages, budgets and finally locations.

Various kinds of transformations, including value transformations, structural and logical transformations, were applied to this initial dataset to generate a set of 80 different test cases. For each test case, the reference alignments are given (i.e. sameAs links between individuals of the generated test case and the ones of the initial dataset). We evaluate the keys here using the first test case (file 1) in which the modifications only concern data property values (typographical errors, lexical variations). Each of the two files contains 1228 descriptions of pairwise distinct film instances, 8401 descriptions of creatures, 2471 descriptions of locations, 67 descriptions of languages and finally 101 different descriptions of budgets concerning the movies.

In this dataset we present only the results of the optimistic heuristic, since the pessimistic does not scale. The number of keys discovered in each of the two files of this dataset exceeds the 30. Thus, we chose to not present them one by one but to focus on the results of linking.

For example, two of the keys found for the class Film are the following the following two keys: \{filmedIn, directedBy, name $\}$, \{estimatedBudget $U$ sed $\}$.

\subsection{Scalability Evaluation}

The complexity of the prefix tree exploration is exponential in terms of the number of the property expression values. In order to test the scalability of our method we have checked experimentally on the seven data sources the benefits of the different kinds of pruning that are used during the prefix tree exploration. More specifically, as it is already mentioned, the pruning that is used in KD2R can be grouped in three categories:

1. Key Inheritance (see section 5.2 (A))

2. NonKey Antimonotonicity (see section 5.2 (B))

3. Key Mononotonicity (see section $5.2(\mathrm{C})$ )

In tables 3 and 4 , we give the results of KD2R in terms of runtime and search space pruning for every data source. The given results correspond to the sum 


\begin{tabular}{|c|c|c|c|c|}
\hline Dataset & Threshold T & \#properties & \#instances & \#triples \\
\hline DBpedia: Person (D4) & $20 \%$ & 7 & 740689 & 2952706 \\
\hline DBpedia: Person (D4) & $10 \%$ & 10 & 742233 & 3332207 \\
\hline DBpedia: NaturalPlace (D5) & $20 \%$ & 11 & 49887 & 836960 \\
\hline
\end{tabular}

Table 2: DBpedia dataset description

of those obtained for each class in the dataset. For example, for the data source s1, the results correspond to the results obtained for the classes Person and Address.

The pruning techniques enable KD2R to be more efficient and scalable in big datasets. Tables 3 and 4 show that on the five smallest data sources, the execution times of keyFinder (using pessimistic or optimistic) is less than 8 seconds. For the two DBpedia data sources, the execution times is less than 441 seconds. Thanks to the different kinds of pruning, less than $50 \%$ of the nodes of the prefix tree are explored for all datasets. Furthermore, we can notice that the more the triples are numerous the more the pruning is efficient. It should be also mentioned that for the instances of the class DBpedia Person, less than 5\% of the nodes are explored, and for the class DBpedia NaturalPlace, less than $0.5 \%$ of the nodes are explored.The dataset D5, is the only one in the experiments that contain sumbsumption relations between the classes. This experiment has been executed to show the importance of the Key inheritance pruning. $13 \%$ of the all the prunings that takes place in this dataset are obtained thanks to the Key Inheritance (4).

Nevertheless, even if the pruning clearly improves the execution time, the bottleneck of the approach is the computation of the minimal keys from the set of maximal non keys and undetermined keys. Indeed, the complexity of this step is quadratic in terms of the number of non keys when the number of keys is linear with respect to the number of non keys and undermined keys.

\subsection{Evaluation of the key quality}

In this section we first present the results, on the IIMB dataset, of the quality evaluation results of the discovered keys using a linking tool without using similarity scores and without propagation. Then we give, for all the other datasets, the linking results using LN2R linking tool where similarity scores and decision propagation are used.

\subsubsection{Key quality without similarity scores and without propagation}

By using a data linking tool where equality is used to compare values and where no propagation between linking decisions is used, we have computed the recall, the precision and the F-measure obtained on a part of the IIMB dataset.

Due to the data heterogeneity of the IIMB dataset, we have obtained a low value for the recall $(5.03 \%)$. Discovering keys that are valid for both datasets allows to guarantee a very high value for the precision. This is shown by the obtained precision which is of $100 \%$. This leads to an F-Measure of about $10 \%$. These results show that the obtained keys has a good quality in terms of correctness. However, due to the heterogeneity of the data and to the fact that decision propagation is not considered the recall is very low. Hence, in order to ensure the good quality results, in terms of recall and precision, more complex linking tools that take similarity measures into account, appear to be necessary to be used.

\subsubsection{Key quality by using similarity scores and deci- sion propagation}

To evaluate the quality of the obtained keys, using a more complex way, we have used an existing data linking tool to show the benefits of using discovered keys in the data linking process. More precisely, we have compared the results that are obtained by the linking tool when the keys that are discovered by KD2R are used and when no keys are not available.

Brief presentation of N2R. N2R is a knowledge based approach which exploits the keys that are declared in the ontology to infer identity links (reconciliation decisions) between class instances.

It exploits keys in order to generate a function that computes similarity scores for pairs of instances. This numerical approach is based on equations that model the influence between similarities. In the equations, each variable represents the (unknown) similarity between two instances while the similarities between values of data properties are constants (obtained using standard similarity measures on strings or on sets of strings). Furthermore, ontology and data knowledge (disjunction, UNA) is exploited by N2R in a filtering step to reduce the number of reference pairs that are considered in the equation system. 


\begin{tabular}{|c|l|l|l|l|l|l|l|}
\hline dataset & source & pruning category & $\begin{array}{l}\text { \#not- } \\
\text { visited- } \\
\text { nodes }\end{array}$ & $\begin{array}{l}\text { not-visited } \\
\text { rate }\end{array}$ & $\begin{array}{l}\text { \#nodes } \\
\text { without } \\
\text { pruning }\end{array}$ & $\begin{array}{l}\text { time } \\
\text { pruning (s) }\end{array}$ & $\begin{array}{l}\text { wime without } \\
\text { pruning (s) }\end{array}$ \\
\hline OAEI Person & $\mathrm{s} 1$ & Key monotonicity & 764478 & $60 \%$ & 1252994 & 4 & \\
\hline OAEI Person & $\mathrm{s} 2$ & Key monotonicity & 1679956 & $75 \%$ & 2234738 & 8 & 8 \\
\hline OAEI Restaurant & $\mathrm{s} 1$ & Key monotonicity & 228 & $81 \%$ & 280 & 1 & 10 \\
\hline OAEI Restaurant & $\mathrm{s} 2$ & Key monotonicity & 103 & $71 \%$ & 146 & 1 & 2 \\
\hline GFT & $\mathrm{s} 1$ & Key monotonicity & 84 & $10 \%$ & 827 & 1 & 3 \\
\hline ChefMoz & $\mathrm{s} 2$ & Key monotonicity & 71754 & $55 \%$ & 129569 & 570 & 625 \\
\hline
\end{tabular}

Table 3: Pessimistic heuristic: search space pruning and runtime results

\begin{tabular}{|c|c|c|c|c|c|c|c|}
\hline dataset & source & pruning category & $\begin{array}{l}\text { \#not- } \\
\text { visited- } \\
\text { nodes }\end{array}$ & $\begin{array}{l}\text { not-visited } \\
\text { rate }\end{array}$ & $\begin{array}{l}\text { \#nodes } \\
\text { without } \\
\text { pruning }\end{array}$ & $\begin{array}{l}\text { time with } \\
\text { pruning }(s)\end{array}$ & $\begin{array}{l}\text { time without } \\
\text { pruning (s) }\end{array}$ \\
\hline OAEI Person & s1 & Key monotonicity & 12156 & $88 \%$ & 13750 & 3 & 7 \\
\hline OAEI Person & s2 & Key monotonicity & 16225 & $89 \%$ & 18276 & 3 & 5 \\
\hline OAEI Restaurant & s1 & Key monotonicity & 228 & $81 \%$ & 280 & 1 & 2 \\
\hline OAEI Restaurant & s2 & Key monotonicity & 103 & $71 \%$ & 146 & 1 & 2 \\
\hline GFT & s1 & Key monotonicity & 108 & $22 \%$ & 499 & 1 & 3 \\
\hline ChefMoz & s2 & Key monotonicity & 27026 & $55 \%$ & 49351 & 5 & 8 \\
\hline DBpedia (Person) & $\mathrm{s} 1(\mathrm{~T}=20 \%)$ & Key monotonicity & 27302986 & $5 \%$ & 28803153 & 441 & 634 \\
\hline \multirow[t]{3}{*}{ DBpedia (NaturalPalce) } & \multirow[t]{3}{*}{ s1 $(\mathrm{T}=20 \%)$} & Key monotonicity & 40907348 & \multirow[t]{3}{*}{$0.5 \%$} & \multirow[t]{3}{*}{47716771} & \multirow[t]{3}{*}{42} & \multirow[t]{3}{*}{222} \\
\hline & & NonKey Antimonotonicity & 159538 & & & & \\
\hline & & Key Inheritance & 6153252 & & & & \\
\hline \multirow[t]{2}{*}{ IIMB Film } & \multirow[t]{2}{*}{ s1 } & Key monotonicity & 223436 & \multirow[t]{2}{*}{$99 \%$} & \multirow[t]{2}{*}{12836301} & \multirow[t]{2}{*}{30} & \multirow[t]{2}{*}{75} \\
\hline & & NonKey Antimonotonicity & 10492012 & & & & \\
\hline \multirow[t]{2}{*}{ IIMB Film } & \multirow[t]{2}{*}{ s2 } & Key monotonicity & 2313411 & \multirow[t]{2}{*}{$98 \%$} & \multirow[t]{2}{*}{9135607} & \multirow[t]{2}{*}{40} & \multirow[t]{2}{*}{82} \\
\hline & & NonKey Antimonotonicity & 6701456 & & & & \\
\hline
\end{tabular}

Table 4: Optimistic heuristic: search space pruning and runtime results 
More precisely, for each reference pair, the similarity score is modeled by a variable $x_{i}$ and the way it depends on other similarity scores is modeled by an equation: $x_{i}=f_{i}(X)$, where $i \in[1 . . n]$ and $n$ is the number of reference pairs for which we apply N2R, and $X=\left(x_{1}, x_{2}, \ldots, x_{n}\right)$ is the set of their corresponding variables. Each equation $x_{i}=f_{i}(X)$ is of the form:

$$
f_{i}(X)=\max \left(f_{i-d f}(X), f_{i-n d f}(X)\right)
$$

The function $f_{i-d f}(X)$ is the maximum of the similarity scores obtained for the instances of the data properties and the object properties that belong to a key describing the $i$-th reference pair. In case of a combined key we compute first the average of the similarity scores of the property instances involved in that combined key. The maximum function allows to propagate the similarity scores of the values and the instances having a strong impact. The function $f_{i-n d f}(X)$ is defined by a weighted average of the similarity scores of the literal value pairs (and sets) and the instance pairs (and sets) of data properties and object properties describing the $i$-th instance pair and not belonging to a key. See [22] for the detailed definition of $f_{i-d f}(X)$ and $f_{i-n d f}(X)$. Solving this equation system is done by an iterative method inspired by the Jacobi method [6], which is fast converging on linear equation systems.

The instance pairs for which the similarity is greater than a given threshold $T R e c$ are reconciled, i.e, an identity link is created between the two instances.

We have compared the obtained results with the available gold-standard using the following standard measures: precision, recall and F-measure. Then, we have compared these results to those that are obtained by N2R: (i) when no keys are declared in the ontology and (ii) when expert keys manually defined for the OAEI' 10 contest are declared in the ontology.

Obtained results on OAEI 2010 datasets. Tables 5 and 6 show the results obtained by N2R in terms of recall, precision and F-measure when: (i) no keys are used, (ii) all KD2R keys are used and (iii) keys defined by experts are used [21]. Since the domains concerning persons and restaurants are rather common, the expert keys have been declared manually by one of the participants of the OAEI contest 2010, for LN2R tool. If several experts are involved, a kappa coefficient [3] can be computed to measure their agreement. Since D1 contains not instantiated properties, both the optimistic and pessimistic heuristic have been performed. In Table 5 we define as $\mathrm{KD} 2 \mathrm{R}-\mathrm{O}$ the results obtained using keys discovered with the optimistic heuristic and KD2R-P the results obtained using key discovered with the pessimistic heuristic. It should be mentioned that for the datasets D2 and D3 the results given for KD2R are both the results of KD2R-O and KD2R-P, since there are no undetermined keys. We show the results when the threshold TRec varies from 1 to 0.8 . Since the F-measure expresses the trade-off between the recall and the precision, we first discuss the obtained results according to this measure. Across all datasets and values of TRec, the F-measure obtained using KD2R keys is greater than the F-Measure obtained when keys are not available. We can notice that, the results obtained for the Person dataset (D1) are better when we use keys obtained by either KD2R-O or KD2R-P than when the keys are not used. When the threshold is bigger than 0.95 the FMeasure of LN2R using KD2R-O keys is $100 \%$. This is an example that shows that the results using keys found with the optimistic heuristic can be better than the ones found with the pessimistic heuristic. In the restaurant dataset (D2), when $T R e c \geq 0.9$, the F-measure is almost three times higher than the F-measure obtained when keys are not declared. This big difference is due to the fact that the recall is much higher when KD2R keys are added. Indeed, even when some property values are syntactically different, it suffices that it exists one key for which the property values are similar, to infer any identity link. For example, when $T R e c=1$, the KD2R recall is $95 \%$ for the persons dataset while without the keys the recall is $0 \%$. Hence, the more numerous the keys are, the more identity links can be inferred.

Furthermore, our results are very close to the ones obtained using expert keys. For both datasets, the largest difference between KD2R F-measure and the expert's one is $6 \%$. We should also mention that $\mathrm{KD} 2 \mathrm{R}$ precision is always higher than the expert precision. Indeed, some expert keys are not verified in the dataset. For example, while the expert has declared phoneNumber as a key for the Restaurant class,in this dataset some restaurants share the same phone number, i.e, they are managed by the same organization.

These results show that the data linking results are significantly improved, especially in terms of recall, when we compare them to results that can be obtained when the keys are not defined.

In table 7 we give a comparison between the results obtained by LN2R using KD2R keys with other tools that have used the Person-Restaurant (PR) dataset of OAEI 2010-Instance Matching track. We can notice that the obtained results in terms of F-measure are comparable to those obtained by semi-supervised approaches like ObjectCoref [8]. It is nevertheless less ef- 


\begin{tabular}{|c|c|c|c|c|}
\hline TRec & Keys & Recall & Precision & F-Measure \\
\hline \multirow{3}{*}{1} & without & $0 \%$ & $-\%$ & $-\%$ \\
\cline { 2 - 5 } & KD2R-O & $100 \%$ & $100 \%$ & $100 \%$ \\
\cline { 2 - 5 } & KD2R-P & $95.00 \%$ & $100 \%$ & $97.44 \%$ \\
\cline { 2 - 5 } & expert & $98.40 \%$ & $100 \%$ & $99.19 \%$ \\
\hline \hline 0.95 & without & $61.20 \%$ & $100 \%$ & $75.93 \%$ \\
\cline { 2 - 5 } & KD2R-O & $100 \%$ & $100 \%$ & $100 \%$ \\
\cline { 2 - 5 } & KD2R-P & $95.00 \%$ & $100 \%$ & $97.44 \%$ \\
\cline { 2 - 5 } & expert & $98.60 \%$ & $100 \%$ & $99.30 \%$ \\
\hline \hline 0.9 & without & $64.2 \%$ & $100 \%$ & $78.20 \%$ \\
\cline { 2 - 5 } & KD2R-O & $100 \%$ & $98.04 \%$ & $99.01 \%$ \\
\cline { 2 - 5 } & KD2R-P & $95.00 \%$ & $100 \%$ & $97.44 \%$ \\
\cline { 2 - 5 } & expert & $98.60 \%$ & $100 \%$ & $99.30 \%$ \\
\hline \hline 0.85 & without & $65.20 \%$ & $100 \%$ & $78.93 \%$ \\
\cline { 2 - 5 } & KD2R-O & $100 \%$ & $81.30 \%$ & $89.68 \%$ \\
\cline { 2 - 5 } & KD2R-P & $99.80 \%$ & $100 \%$ & $99.90 \%$ \\
\cline { 2 - 5 } & expert & $99.80 \%$ & $100 \%$ & $99.90 \%$ \\
\hline \hline 0.8 & without & $90.20 \%$ & $100 \%$ & $94.85 \%$ \\
\cline { 2 - 5 } & KD2R-O & $100 \%$ & $35.71 \%$ & $52.63 \%$ \\
\cline { 2 - 5 } & KD2R-P & $99.80 \%$ & $100 \%$ & $99.90 \%$ \\
\cline { 2 - 5 } & expert & $100 \%$ & $100 \%$ & $100 \%$ \\
\hline
\end{tabular}

\begin{tabular}{|c|c|c|c|c|}
\hline TRec & Keys & Recall & Precision & F-Measure \\
\hline \multirow{2}{*}{1} & without & $45.67 \%$ & $100 \%$ & $62.71 \%$ \\
\cline { 2 - 5 } & KD2R & $60.49 \%$ & $100 \%$ & $75.38 \%$ \\
\hline \hline \multirow{2}{*}{0.95} & without & $50.61 \%$ & $100 \%$ & $67.21 \%$ \\
\cline { 2 - 5 } & KD2R & $60.49 \%$ & $100 \%$ & $75.38 \%$ \\
\hline \hline \multirow{2}{*}{0.9} & without & $50.61 \%$ & $100 \%$ & $67.21 \%$ \\
\cline { 2 - 5 } & KD2R & $60.49 \%$ & $100 \%$ & $75.38 \%$ \\
\hline \hline \multirow{2}{*}{0.85} & without & $50.61 \%$ & $100 \%$ & $67.21 \%$ \\
\cline { 2 - 5 } & KD2R & $60.49 \%$ & $100 \%$ & $75.38 \%$ \\
\hline \hline \multirow{2}{*}{0.8} & without & $54.32 \%$ & $100 \%$ & $70.39 \%$ \\
\cline { 2 - 5 } & KD2R & $60.49 \%$ & $100 \%$ & $75.38 \%$ \\
\hline \hline 0.75 & without & $54.32 \%$ & $100 \%$ & $70.39 \%$ \\
\cline { 2 - 5 } & KD2R & $60.49 \%$ & $100 \%$ & $75.38 \%$ \\
\hline \hline \multirow{2}{*}{0.7} & without & $60.49 \%$ & $100 \%$ & $75.38 \%$ \\
\cline { 2 - 5 } & KD2R & $61.72 \%$ & $100 \%$ & $76.33 \%$ \\
\hline
\end{tabular}

Table 8: Recall, Precision and F-measure for D3

sults when the threshold TRec takes values in the interval [0.7..1]. For both datasets and for every $T R e c$ value, the F-measure found using KD2R keys is greater than the F-Measure when keys are missing.

This difference is due to the fact that the recall is always higher when KD2R keys are added. Indeed, even when some property values are syntactically different, it suffices that it exists one key for which the property values are similar, to infer the reconciliation. For example, when $T R e c=1$, the KD2R recall is $60 \%$ for the persons dataset while without the keys the recall is $45 \%$. Hence, the more numerous the keys are, the more reconciliation decisions can be inferred.

As in D1 and D2, the above results show that the data linking results are significantly improved, in particular in terms of recall, when we compare them to results obtained when the keys are not defined.

\section{Related Work}

The problem of key discovery in RDF datasets in the setting of the semantic web is similar to the key discovery problem in relational databases. Nevertheless, in database area the approaches do not consider the semantics defined in the ontology (e.g. the subsumption relation that can be defined between classes). Besides, in the relational context, the key discovery problem

is a sub-problem of Functional Dependencies (FDs)

ficient than approaches that learn linkage rules that are specific to the dataset like KoFuss+GA.

Obtained results for GFT-ChefMoz dataset. Table 8 demonstrates the results obtained by N2R in terms of recall, precision and F-measure when: (i) no keys are used and (ii) KD2R keys are used. We show the re- 


\begin{tabular}{|c|c|c|c|c|c|c|c|c|}
\hline Dataset & LN2R+KD2R-P & LN2R+KD2R-O & ASMOV & LN2R & CODI & ObjectCoref & RIMOM & KnoFuss+GA \\
\hline Person 1 & 0.99 & 1.00 & 1.00 & 1.00 & 0.91 & 1.00 & 1.00 & 1.00 \\
\hline Restaurant & 0.728 & - & 0.70 & 0.75 & 0.72 & 0.73 & 0.81 & 0.78 \\
\hline
\end{tabular}

Table 7: Comparison of F-Measure with other tools on PR dataset of OAEI 2010 benchmark

discovery from data. Indeed, a FD states that the value of one attribute is uniquely determined by the values of some other attributes.

Keys or FDs can be used for different purposes. Some approaches focus on finding approximate keys or FDs. Blocking methods aim at using approximate keys to reduce the number of instance pairs that have to be compared by a data linking tool ([13],[24]). In [24], discriminating data type properties (i.e approximate keys) are discovered from a dataset. Then, only the instance pairs that have similar litteral values for these discriminating properties are selected. These properties are chosen using unsupervised learning techniques and keys of size $n$ are explored only if there is no key of size $n-1$ with a discriminative power higher enough. In more details, the aim here is to find the best approximate keys to construct blocks of instances and not to discover the largest set of valid minimal keys that can be used to link data. Other approaches use approximate keys to infer probable identity links. In [25], the authors discover (inverse) functional properties from data sources where the UNA is fullfilled (i.e. non composite keys). The functionality degree of a property is computed to generate probable identity links. More precisely, for one instance, the local functionality degree of a property is the number of distinct values (or instances) that are the object of the property when the considered instance is the subject. The functionality degree of one property is the harmonic mean of the local functionality degrees across all the instances; the inverse functionality degree is defined analogously. In a data mining setting, the framework defined by [12] can be used to discover approximate keys. In this approach, a levelwise algorithm starts from the longuest keys and the partial order that can be defined between keys is used to avoid exploring subsets of non keys.

Functional dependencies can be used in reverse engineering, query optimization or for data mining purposes. [29] proposes a way of retrieving non composite probabilistic FDs from a set of data sources. Two strategies are proposed: the first merges the data before discovering FDs, while the second merges the FDs obtained from each data source. In order to find the approximated FDs that hold in a relation,
TANE [9] partitions the tuples into groups based on their attribute values. When the size of the partition is 1 , the partition is eliminated based on the fact that its data cannot represent counter-examples of more complex functional dependencies, so the partition is eliminated. In this work, the FD is associated to an error measure which is the minimal fraction of tuples to remove for the key to hold in the dataset. In [2], the authors have developed an approach based on TANE [9] algorithm to discover pseudo-keys for which a few instances may have the same values for the properties of a key. In all these aproaches, to compute the confidence degree or the error measure that can be associated to a key or a FD, all the data have to be explored.

Other approaches aim to enrich the ontology and/or use the keys to generate identity links between pairs of instances that can be propagated to other pairs of instances $([22,1])$. Such approaches are called collective or global approaches of data linking. For example, if the approach can find that two paintings are the same, then their museums can be linked and this link will lead to generate identity links between the cities where the museums are located in. Other approaches, such as [31] discover keys or semantic dependencies to detect erroneous data. For these kinds of approaches, only keys that are as correct as possible (i.e. valid with regard to the dataset) are useful.

In the context of Open Linked Data, [17] have proposed a supervised approach to learn (inverse) functional properties on a set of reconciled data.

In the relational context, the Gordian method [23] allows discovering composite keys that can be used in tasks related to data integration, anomaly detection, query formulation, query optimization, or indexing. In order to avoid checking all the possible combinations of candidate keys, the method discovers first the maximal non-keys and use them to derive the minimal keys. To optimize the prefix tree exploration, this method exploits the anti-monotonicity property of a non key. Nevertheless, it is assumed that the data are completely described (without null values). Furthermore, multivalued attributes are not taken into account.

KD2R aims to discover keys that are correct with regard to a set of data sources. The approach does not 
need training data and exploits data sources where the UNA is fullfilled. One important feature of KD2R is that it can discover composite keys. Indeed, non composite keys are not frequent in real datasets, and the more numerous the keys are, the more the number of decisions increases. Furthermore, KD2R do not need to explore all the data for each property expression combination. Unlike [2], KD2R do not discover approximate keys (pseudo-keys) that allows few redundancies. However, KD2R distinguishes keys from undetermined keys when not instantiated properties prevent the system to decide whether a set of properties is a key or not. Since the approach is defined in the setting of the semantic Web, it takes into account the subsumption relation defined between classes, multivaluations and incomplete data.

$[16,10,15]$ discover expressive linkage rules which specify the conditions two data items must fulfill to be linked: data transformations, similarity measures, thresholds and the aggregation function. These rules are learnt on a set of existing links [10, 15] or on a dataset where UNA is fulfilled [16] using genetic programming techniques. These rules are specific to the vocabulary used in the datasets while keys do not take into account such kind of information. Keys express conceptual knowledge that can be used either to infer identity links logically or to generate similarity functions as in [22].

\section{Conclusions and Future Work}

In this paper, we have described the method KD2R which aims to discover keys in RDF data in order to use them in data linking. These data conform to distinct ontologies that are aligned and are described in RDF files for which the UNA is fulfilled. KD2R takes into account the properties that the RDF files may have: incompleteness and multi-valuation. Since the data may be numerous, the method discovers maximal undetermined/non keys that are used to compute keys and merge them if keys are discovered from different datasets. Furthermore, the approach exploits key inheritance due to subsumption relations between classes to prune the key search for a given class.

The experiments have been conducted on five datasets. Two datasets have been used in OAEI evaluation initiative and three datasets have been collected from the Web of data. These experiments showed that the use of KD2R keys improves significantly the results obtained by a knowledge-based data linking method, in terms of recall. Furthermore, the experiments showed the scalability of KD2R as it is capable of handling big datasets that contain millions of triples.

In the future, we plan to perform additional experiments in order to show the generality of the approach when applied on other application domains. Moreover, we will study heuristics that determine the best order of the property expressions in the prefix tree. It would be also interesting to study more deeply the automatic key discovery problem when the UNA is not fulfilled and/or the data contain errors. This can be done by relaxing the validity constraint and finding keys where exceptions are allowed. Therefore, KD2R approach will be extended to find maximal non keys having at least $\alpha$ (a threshold fixed by the user) redundancies. Those maximal non-keys will be used to derive keys that are valid with $\beta$ exceptions. Finally, we are interested in studying how paths of property expressions, that can uniquely identify an entity, can be automatically discovered.

\section{Acknowledgment}

This work is supported by the French National Research Agency: "Quality and Interoperability of Large Catalogues of Documents" project (QUALINCA-ANR-2012-CORD-01202).

\section{References}

[1] Arasu, A., Ré, C., Suciu, D., 2009. Large-scale deduplication with constraints using dedupalog, in: ICDE, pp. 952-963.

[2] Atencia, M., David, J., Scharffe, F., 2012. Keys and pseudokeys detection for web datasets cleansing and interlinking, in: EKAW, pp. 144-153.

[3] Cohen, J., 1968. Weighted kappa: nominal scale agreement with provision for scaled disagreement or partial credit. Psychological Bulletin 70, 213-220.

[4] Elmagarmid, A.K., Ipeirotis, P.G., Verykios, V.S., 2007. Duplicate record detection: A survey. IEEE Transactions on Knowledge and Data Engineering 19, 1-16.

[5] Ferrara, A., Nikolov, A., Scharffe, F., 2011. Data linking for the semantic web. Int. J. Semantic Web Inf. Syst. 7, 46-76.

[6] Golub, G.H., Loan, C.F.V., 1996. Matrix computations (3rd ed.). Johns Hopkins University Press, Baltimore, MD, USA.

[7] Gonzalez, H., Halevy, A.Y., Jensen, C.S., Langen, A., Madhavan, J., Shapley, R., Shen, W., Goldberg-Kidon, J., 2010. Google fusion tables: web-centered data management and collaboration, in: SIGMOD Conference, pp. 1061-1066.

[8] Hu, W., Chen, J., Qu, Y., 2011. A self-training approach for resolving object coreference on the semantic web, in: WWW, pp. 87-96.

[9] Huhtala, Y., Kärkkäinen, J., Porkka, P., Toivonen, H., 1999. Tane: An efficient algorithm for discovering functional and approximate dependencies. The Computer Journal 42, 100-111.

[10] Isele, R., Bizer, C., 2012. Learning expressive linkage rules using genetic programming. PVLDB 5, 1638-1649.

[11] Low, W.L., Lee, M.L., Ling, T.W., 2001. A knowledge-based approach for duplicate elimination in data cleaning. Information Systems 26, 585-606. 
[12] Mannila, H., Toivonen, H., 1997. Levelwise search and borders of theories in knowledge discovery. Data Min. Knowl. Discov. 1, 241-258.

[13] Michelson, M., Knoblock, C.A., 2006. Learning blocking schemes for record linkage, in: AAAI, pp. 440-445.

[14] Ngomo, A.C.N., Auer, S., 2011. Limes a time-efficient approach for large-scale link discovery on the web of data, in: IJCAI, pp. 2312-2317.

[15] Ngomo, A.C.N., Lyko, K., 2012. Eagle: Efficient active learning of link specifications using genetic programming, in: 9th Extended Semantic Web Conference (ESWC), pp. 149-163.

[16] Nikolov, A., d'Aquin, M., Motta, E., 2012. Unsupervised learning of link discovery configuration, in: 9th Extended Semantic Web Conference (ESWC), Springer-Verlag, Berlin, Heidelberg. pp. 119-133.

[17] Nikolov, A., Motta, E., 2010. Data linking: Capturing and utilising implicit schema-level relations, in: Proceedings of Linked Data on the Web workshop at 19th International World Wide Web Conference(WWW)'2010.

[18] Patel-Schneider, P.F., Hayes, P., Horrocks, I., 2004. OWL Web Ontology Language Semantics and Abstract Syntax Section 5. RDF-Compatible Model-Theoretic Semantics. Technical Report. W3C.

[19] Pavel, S., Jerome, E., 2013. Ontology matching: state of the art and future challenges. IEEE Transactions on knowledge and data engineering 5, 158-176.

[20] Quercini, G., Setz, J., Sonntag, D., Reynaud, C., 2012. Facetted browsing on extracted fusion tables data for digital cities, in: proceedings of the Web of Linked Entities (WoLE) workshop in conjonction with ISWC 2012 conference, pp. 94-105.

[21] Saïs, F., Niraula, N.B., Pernelle, N., Rousset, M.C., 2010. LN2R a knowledge based reference reconciliation system: Oaei 2010 results, in: Proceedings of the 5th International Workshop on Ontology Matching (OM-2010).

[22] Saïs, F., Pernelle, N., Rousset, M.C., 2009. Combining a logical and a numerical method for data reconciliation. Journal on Data Semantics 12, 66-94.

[23] Sismanis, Y., Brown, P., Haas, P.J., Reinwald, B., 2006. Gordian: efficient and scalable discovery of composite keys, in: Proceedings of the 32nd International conference Very Large Data Bases (VLDB), VLDB Endowment. pp. 691-702.

[24] Song, D., Heflin, J., 2011. Automatically generating data linkages using a domain-independent candidate selection approach, in: Proceedings of the 10th International Semantic Web Conference(ISWC) - Volume Part I, Springer-Verlag, Berlin, Heidelberg. pp. 649-664.

[25] Suchanek, F.M., Abiteboul, S., Senellart, P., 2011. Paris: Probabilistic alignment of relations, instances, and schema. The Proceedings of the VLDB Endowment(PVLDB) 5, 157-168.

[26] Suchanek, F.M., Kasneci, G., Weikum, G., 2007. Yago: a core of semantic knowledge, in: Proceedings of the 16th International Conference on World Wide Web (WWW), pp. 697-706.

[27] Symeonidou, D., Pernelle, N., Saïs, F., 2011. KD2R : a Key Discovery method for semantic Reference Reconcilation, in: 7th International IFIP Workshop on Semantic Web \& Web Semantics (SWWS 2011), Springer Verlag, Heraklion, Greece. pp. 392401.

[28] Volz, J., Bizer, C., Gaedke, M., Kobilarov, G., 2009. Discovering and maintaining links on the web of data, in: Proceedings of the 8th International Semantic Web Conference(ISWC), Springer-Verlag, Berlin, Heidelberg. pp. 650-665.

[29] Wang, D.Z., Dong, X.L., Sarma, A.D., Franklin, M.J., Halevy, A.Y., 2009. Functional dependency generation and applications in pay-as-you-go data integration systems, in: WebDB.

[30] Winkler, W.E., 2006. Overview of record linkage and current research directions. Technical Report. Bureau of the Census.

[31] Yu, Y., Li, Y., Heflin, J., 2011. Detecting abnormal semantic web data using semantic dependency, in: Proceedings of the 2011 IEEE Fifth International Conference on Semantic Computing, IEEE Computer Society, Washington, DC, USA. pp. 154157. 